\title{
Uniform attractors for non-autonomous wave equations with nonlinear damping
}

\author{
Chunyou Sun, Daomin Cao \\ Institute of Applied Mathematics, Chinese Academy of Sciences \\ Beijing, 100080, China \\ E-mails: cysun@amss.ac.cn; dmcao@amt.ac.cn \\ and \\ Jinqiao Duan \\ Department of Applied Mathematics, Illinois Institute of Technology \\ Chicago, IL 60616, USA \\ E-mail: duan@iit.edu
}

\begin{abstract}
We consider dynamical behavior of non-autonomous wave-type evolutionary equations with nonlinear damping, critical nonlinearity, and time-dependent external forcing which is translation bounded but not translation compact (i.e., external forcing is not necessarily time-periodic, quasi-periodic or almost periodic). A sufficient and necessary condition for the existence of uniform attractors is established using the concept of uniform asymptotic compactness. The required compactness for the existence of uniform attractors is then fulfilled by some new a priori estimates for concrete wave type equations arising from applications. The structure of uniform attractors is obtained by constructing a skew product flow on the extended phase space for the norm-to-weak continuous process.
\end{abstract}

Keywords: Non-autonomous systems; Wave equation; Nonlinear damping; Critical exponent; Uniform attractor; Norm-to-weak continuous process.

Date: June 26, 2006.

AMS Subject Classification: 35L05, 35B40, 35B41

This work was partly supported by the NSF Grants DMS-0209326 \& DMS-0542450, and the Outstanding Overseas Chinese Scholars Fund of the Chinese Academy of Sciences. 


\section{Introduction}

We consider the following non-autonomous wave equations with nonlinear damping, on a bounded domain $\Omega$ in $\mathbb{R}^{3}$ with smooth boundary:

$$
u_{t t}+h\left(u_{t}\right)-\Delta u+f(u)=g(x, t) \quad x \in \Omega
$$

under the boundary condition

$$
\left.u\right|_{\partial \Omega}=0
$$

and initial conditions

$$
u(x, 0)=u_{0}(x), \quad u_{t}(x, 0)=v_{0}(x) .
$$

Here $h$ is the nonlinear damping function, $f$ is the nonlinearity, and $g$ is a given external time-dependent forcing.

Equation (1.1) arises as an evolutionary mathematical model in various systems, for example: (i) modeling a continuous Josephson junction with specific $h, g$ and $f$; (ii) modeling a hybrid system of nonlinear waves and nerve conduct; and(iii) modeling a phenomenon in quantum mechanics. A relevant physical issue is to investigate the asymptotic dynamical behavior of these mathematical models.

For the special, autonomous case of (1.1), i.e., when $g$ does not depend on time $t$ explicitly, the solution operator defines a flow or semigroup. The asymptotic behaviors of the solutions have been studied extensively by using of the concept of global attractors; see, for example, 11, 2, 3, 11, 21, 38, for the linear damping case, and [12, 13, 14, 19, 20, 36. for the nonlinear damping case.

In the general case of non-autonomous system (1.1), the solution operator does not define a flow or semigroup, but a process; see Section 2 and Section 5 below. A proper extension of the notion of a global attractor for semigroups to the case of processes is the so-called uniform attractor (see e.g. [23, 9, 11]). About the basic concepts of nonautonomous dynamical systems, uniform attractors and processes, we refer to [23, 9, 11] for more details or see Section 2 below.

The basic assumptions about nonlinear damping $h$ and nonlinearity $f$ are as follows:

$$
\begin{gathered}
h \in C^{1}(\mathbb{R}), \quad h(0)=0, \quad h \text { is strictly increasing, } \\
\liminf _{|s| \rightarrow \infty} h^{\prime}(s)>0, \\
|h(s)| \leq C_{1}\left(1+|s|^{p}\right),
\end{gathered}
$$


where $p \in[1,5) ; f \in C^{1}(\mathbb{R})$ and satisfies

$$
\begin{gathered}
\left|f^{\prime}(s)\right| \leq C_{2}\left(1+|s|^{q}\right), \\
\liminf _{|s| \rightarrow \infty} \frac{f(s)}{s}>-\lambda_{1},
\end{gathered}
$$

where $0 \leqslant q \leqslant 2$ and $\lambda_{1}$ is the first eigenvalue of $-\Delta$ in $H_{0}^{1}(\Omega)$, and these assumptions are similar to that for autonomous system and come from [12, 13, 19] etc.

In this paper, we consider the non-autonomous system (1.1)-(1.3) via the uniform attractors of the corresponding family of processes $\left\{U_{\sigma}(t, \tau)\right\}, \sigma \in \Sigma$, especially with: (i) the nonlinear damping (i.e., $h$ is a nonlinear function), (ii) the nonlinearity $f(u)$ has critical exponent $(q=2)$, and (iii) the external forcing $g(x, t)$ is not translation compact in $L_{\text {loc }}^{2}\left(\mathbb{R} ; L^{2}(\Omega)\right)$.

In Chepyzhov \& Vishik [1], for the linear damping case $h(v)=k v$ with a constant $k>0$ and $q<2$ (subcritical), for system (1.1)-(1.3), the authors obtained the existence of a bounded uniform absorbing set if $g$ is translation bounded, and the existence of a uniformly attractor when $g$ is translation compact (e.g., when $g$ is time-periodic, quasiperiodic or almost periodic). Under the assumptions that $g$ and $\partial_{t} g$ are both in the space of bounded continuous functions $C_{b}\left(\mathbb{R}, L^{2}(\Omega)\right)$ and $h$ satisfies the growth bounds $0<\alpha \leqslant h^{\prime}(s) \leqslant \beta<\infty$ for some constants $\alpha$ and $\beta$, Zhou \& Wang 41] have proved the existence of kernel sections and obtained the estimation of the Hausdorff dimension of the kernel sections.

For the existence of uniform attractors, as in autonomous case, some kind of compactness of the family of processes is a key ingredient. The corresponding compactness assumption in [23, 9, 11] is that the family of processes $\left\{U_{\sigma}(t, \tau)\right\}, \sigma \in \Sigma$ has a compact uniformly absorbing set. The number $q=2$ is called the critical exponent, since the nonlinearity $f$ is not compact in this case, which is an essential difficulty in studying the asymptotic behavior even for the autonomous case [1, 2, 3, 12, 13, 19, 36] etc.

About the case of $1<p<5$ for the nonlinear damping exponent $p$, as mentioned in Haraux [22, even for the bounded dissipation, it becomes much more difficult when $g$ depends on $t$, and the characterizations of dynamics for this case are unknown to the authors. Moreover, the nonlinearity of $h$ also brings some difficulties for us to prove the compactness, for example, for the autonomous linearly damped wave equations, Ball [2] gives a very nice method to verify the necessary asymptotic compactness, so-called energy methods by many other authors, and then this method is generalized and given a general abstract framework for its applications by [28, 32, 33] and others to both autonomous and 
non-autonomous cases. However, for our problem, due to the nonlinear damping, it seems to be difficult to apply the method of Ball [2].

The purpose of this paper is to obtain the existence and structure of the compact, in the norm topology of $H_{0}^{1} \times L^{2}$, uniform attractor when the external forcing $g(x, t)$ is not translation compact in $L_{l o c}^{2}\left(\mathbb{R} ; L^{2}(\Omega)\right)$. For the existence of uniform attractors, a main approach in [9, 11] is by constructing skew product flow on the extend phase space $X \times \Sigma$. They require that the symbol space has some compactness so that the skew product flow has some corresponding compactness, i.e., the concept of translation compact functions (e.g., see [9, 10, 11]). Consequently, the compact uniform attractors are obtained for the systems with symbols of compact hulls, and the weakly compact uniform attractors for the systems with symbols of weakly compact hulls. However, there are some results show that one can obtain the compact uniform attractors for the system with translation noncompact external forcing: by generalizing the methods in [30, the authors in [29] obtain the existence of an uniform attractor for 2D Navier-Stokes equation in bounded domain with a kind of translation noncompact external forcing; in Zelik 39], by use of a bootstrap argument together with a sharp use of Gronwall-type lemmas, when $h(v) \equiv k v$ and $g, \partial_{t} g \in L^{\infty}\left(\mathbb{R} ; L^{2}(\Omega)\right)$, the author obtains some regularity estimates for the solutions of (1.1), which implies naturally the existence of an uniform attractor; see also the results in Chepyzhov \& Vishik [8].

Furthermore, we consider the structure of the uniform attractor by investigating the kernel sections of a process (see 9, 11] for more details).

Here, for system (1.1)-(1.3), we further assume that

$$
g(\cdot, t) \in L^{\infty}\left(\mathbb{R} ; L^{2}(\Omega)\right)
$$

and

$$
\partial_{t} g \in L_{b}^{r}\left(\mathbb{R} ; L^{r}(\Omega)\right) \text { with } r>\frac{6}{5},
$$

where the space $L_{b}^{r}\left(\mathbb{R} ; L^{r}(\Omega)\right)$ of "translation bounded" functions will be defined in the beginning of the next section. Roughly speaking, the two conditions (1.9)- (1.10) mean that the external forcing $g$ is bounded in time and its time-derivative $\partial_{t} g$ is translation bounded. It is clear that a function $g$ satisfies (1.9) and (1.10) dose not need to be translation compact in $L_{l o c}^{2}\left(\mathbb{R} ; L^{2}(\Omega)\right)$. Moreover, we remark that the technical hypothesis (1.9) is mainly for the existence of a bounded uniformly absorbing set; see Theorem 5.3 below or [22] for more details.

It is interesting to note that if (1.10) is replaced by the assumption that $g$ is translation compact (e.g., $g$ is a periodic, quasi-periodic or almost periodic function in $L_{\text {loc }}^{2}\left(\mathbb{R} ; L^{2}(\Omega)\right)$ ), 
then our result on uniform attractors (see Theorem 5.12) in Section 5 still holds, but the proof can be largely simplified; see (5.28)-(5.29), Remark 5.11] and Remark 5.13 below. At the same time, the method in [29] can not be applied to our problem as (1.1) is a hyperbolic type of equation, and the decomposition \& regularization method in [8, 39] appear also not applicable here due to the nonlinearity of $h$.

This paper is organized as follows. After introducing some basic materials in Section 2 , we first present a criterion for the existence of a compact uniform attractor in Section 3 , using the concept of uniform asymptotic compactness (different from the corresponding concept in [9, 11]) which is introduced by Moise et al in 32 for the family of semi-processes. We apply this concept to the family of processes; see Definition 3.1 and Theorem 3.4 Then, we investigate the structure of the uniform attractor via kernel sections of a process. In fact, we present results on uniform attractors and their decompositions into kernel sections for norm-to-weak continuous processes (see Definition 3.5. Theorem 3.8 and Theorem 3.10). Note that the norm-to-weak continuity here is weaker than the usual norm-to-norm and weak-to-weak continuities [40].

In Section 4, partially inspired by the results in [12, 13, 14, 24, 37, we present a simple method for verifying the uniform asymptotic compactness for processes generated by wave type evolutionary equations like (1.1); see Theorem 4.2

Finally, in Section 5, as applications to concrete wave type evolutionary equations, we first prove the existence of compactly uniform (w.r.t $\sigma \in \Sigma$ ) attractors when the external forcing $g_{0}=\sigma_{0}$ satisfies (1.9) and (1.10); see Theorem [5.12, then by verifying the normto-weak continuity we show that the uniform attractor w.r.t. initial time $\tau$ of a process $\left\{U_{\sigma_{0}}(t, \tau)\right\}$ coincides with the uniform attractor w.r.t. symbol $\sigma \in \Sigma^{\prime}$ when the external forcing $g_{0}=\sigma_{0} \in W^{1, \infty}\left(\mathbb{R} ; L^{2}(\Omega)\right)$; we further decompose this uniform attractor into kernel sections; see Lemma 5.16 and Theorem 5.17

\section{Preliminaries}

In this section, we recall some basic concepts about non-autonomous systems. We refer to [23, 9, 11] and the references therein for more details.

Space of translation bounded functions in $L_{l o c}^{r}\left(\mathbb{R} ; L^{k}(\Omega)\right)$, with $r, k \geqslant 1$ :

$$
L_{b}^{r}\left(\mathbb{R} ; L^{k}(\Omega)\right)=\left\{g \in L_{l o c}^{r}\left(\mathbb{R} ; L^{k}(\Omega)\right): \sup _{t \in \mathbb{R}} \int_{t}^{t+1}\left(\int_{\Omega}|g(x, t+s)|^{k} d x\right)^{\frac{r}{k}} d s<\infty\right\} ;
$$


Space of translation compact functions in $L_{l o c}^{2}\left(\mathbb{R} ; L^{2}(\Omega)\right)$ :

$$
\begin{aligned}
L_{c}^{2}\left(\mathbb{R} ; L^{2}(\Omega)\right)=\{ & g \in L_{\text {loc }}^{2}\left(\mathbb{R} ; L^{2}(\Omega)\right): \text { For any interval }\left[t_{1}, t_{2}\right] \subset \mathbb{R}, \\
& \left.\{g(x, h+s): h \in \mathbb{R}\} \mid\left[t_{1}, t_{2}\right] \text { is precompact in } L^{2}\left(t_{1}, t_{2} ; L^{2}(\Omega)\right)\right\} .
\end{aligned}
$$

Let $X$ be a complete metric space and $\Sigma$ be a parameter set.

The operators $\left\{U_{\sigma}(t, \tau)\right\}, \sigma \in \Sigma$ are said to be a family of processes in $X$ with symbol space $\Sigma$ if for any $\sigma \in \Sigma$

$$
\begin{gathered}
U_{\sigma}(t, s) \circ U_{\sigma}(s, \tau)=U_{\sigma}(t, \tau), \quad \forall t \geqslant s \geqslant \tau, \tau \in \mathbb{R}, \\
U_{\sigma}(\tau, \tau)=I d \text { (identity), } \forall \tau \in \mathbb{R} .
\end{gathered}
$$

Let $\{T(s)\}_{s \geqslant 0}$ be the translation semigroup on $\Sigma$, we say that a family of processes $\left\{U_{\sigma}(t, \tau)\right\}, \sigma \in \Sigma$ satisfies the translation identity if

$$
\begin{gathered}
U_{\sigma}(t+s, \tau+s)=U_{T(s) \sigma}(t, \tau), \quad \forall \sigma \in \Sigma, t \geqslant \tau, \tau \in \mathbb{R}, s \geqslant 0, \\
T(s) \Sigma=\Sigma, \quad \forall s \geqslant 0 .
\end{gathered}
$$

By $\mathcal{B}(X)$ we denote the collection of the bounded sets of $X$, and $\mathbb{R}^{\tau}=\{t \in \mathbb{R}, t \geqslant \tau\}$.

Definition 2.1. [11] $A$ bounded set $B_{0} \in \mathcal{B}(X)$ is said to be a bounded uniformly (w.r.t. $\sigma \in \Sigma$ ) absorbing set for $\left\{U_{\sigma}(t, \tau)\right\}, \sigma \in \Sigma$ if for any $\tau \in \mathbb{R}$ and $B \in \mathcal{B}(X)$ there exists $T_{0}=T_{0}(B, \tau)$ such that $\bigcup_{\sigma \in \Sigma} U_{\sigma}(t ; \tau) B \subset B_{0}$ for all $t \geqslant T_{0}$.

Definition 2.2. [11] $A$ set $A \subset X$ is said to be uniformly (w.r.t $\sigma \in \Sigma$ ) attracting for the family of processes $\left\{U_{\sigma}(t, \tau)\right\}, \sigma \in \Sigma$ if for any fixed $\tau \in \mathbb{R}$ and any $B \in \mathcal{B}(X)$

$$
\lim _{t \rightarrow+\infty}\left(\sup _{\sigma \in \Sigma} \operatorname{dist}\left(U_{\sigma}(t ; \tau) B ; A\right)\right)=0,
$$

here $\operatorname{dist}(\cdot, \cdot)$ is the usual Hausdorff semidistance in $X$ between two sets.

In particular, a closed uniformly attracting set $\mathscr{A}_{\Sigma}$ is said to be the uniform (w.r.t. $\sigma \in \Sigma)$ attractor of the family of processes $\left\{U_{\sigma}(t, \tau)\right\}, \sigma \in \Sigma$ if it is contained in any closed uniformly attracting set (minimality property).

Obviously, if the uniform (w.r.t. $\sigma \in \Sigma$ ) attractor exists, it is unique.

In order to obtain the structure as well as the existence of the uniform attractor, under the condition (2.3)-(2.4), the authors in [1] construct the skew product flow in $X \times \Sigma$ :

$$
S(t)(u, \sigma)=\left(U_{\sigma}(t, 0) u, T(t) \sigma\right), \quad t \geqslant 0,(u, \sigma) \in X \times \Sigma,
$$

and $\{S(t)\}_{t \geqslant 0}$ forms a semigroup on $X \times \Sigma$. 


\section{Abstract results}

\subsection{Existence of the uniform attractor}

In this subsection, we present a criterion for the existence of compact uniform attractor using the concept of uniform (w.r.t. $\sigma \in \Sigma$ ) asymptotical compactness, which is different from the corresponding concept in 9, 11, and it is introduced in Moise et al 32 for a family of semi-processes. Now, we use this concept to the family of processes.

Definition 3.1. [32] A family of processes $\left\{U_{\sigma}(t, \tau)\right\}, \sigma \in \Sigma$ on a complete metric space $X$ is said to be uniformly (w.r.t. $\sigma \in \Sigma$ ) asymptotically compact, if and only if for any fixed $\tau \in \mathbb{R}$, bounded sequence $\left\{u_{n}\right\}_{n=1}^{\infty} \subset X,\left\{\sigma_{n}\right\}_{n=1}^{\infty} \subset \Sigma$ and any $\left\{t_{n}\right\}_{n=1}^{\infty} \subset \mathbb{R}^{\tau}$ with $t_{n} \rightarrow \infty$ as $n \rightarrow \infty$, sequence $\left\{U_{\sigma_{n}}\left(t_{n}, \tau\right) u_{n}\right\}_{n=1}^{\infty}$ is precompact in $X$.

Similarly, define the uniform $\omega$-limit set of $B \subset X$ at initial time $\tau$ by

$$
\omega_{\tau, \Sigma}(B)=\bigcap_{t \geqslant \tau} \overline{\bigcup_{\sigma \in \Sigma} \bigcup_{s \geqslant t} U_{\sigma}(s, \tau) B},
$$

where $\bar{A}$ means the closure of $A$ in $X$.

Then, we have the following characterizations for the uniform $\omega$-limit set, see [11, 32,

Proposition 3.2. For any bounded set $B \subset \mathcal{B}(X), u \in \omega_{\tau, \Sigma}(B)$ if and only if there exist $\left\{u_{n}\right\}_{n=1}^{\infty} \subset B,\left\{\sigma_{n}\right\}_{n=1}^{\infty} \subset \Sigma$ and $\left\{t_{n}\right\}_{n=1}^{\infty} \subset \mathbb{R}^{\tau}$ with $t_{n} \rightarrow \infty$ as $n \rightarrow \infty$ such that $U_{\sigma_{n}}\left(t_{n}, \tau\right) u_{n} \rightarrow u$.

In the following, similar to [29], we give some characterizations for the uniform (w.r.t. $\sigma \in \Sigma)$ asymptotically compact processes.

Lemma 3.3. Let $\left\{U_{\sigma}(t, \tau)\right\}, \sigma \in \Sigma$ be a family of uniform (w.r.t. $\sigma \in \Sigma$ ) asymptotically compact processes on a complete metric space $X$, then for any $\tau \in \mathbb{R}$ and any nonempty set $B \in \mathcal{B}(X)$, we have

(i) $\omega_{\tau, \Sigma}(B)$ is nonempty and compact in $X$;

(ii) $\lim _{t \rightarrow+\infty} \sup _{\sigma \in \Sigma} \operatorname{dist}\left(U_{\sigma}(t, \tau) B, \omega_{\tau, \Sigma}(B)\right)=0$;

(iii) if $Y$ is closed and uniform (w.r.t. $\sigma \in \Sigma$ ) attracts $B$, then $\omega_{\tau, \Sigma}(B) \subset Y$;

furthermore, if $\left\{U_{\sigma}(t, \tau)\right\}, \sigma \in \Sigma$ satisfies the translation identity (2.3)-(2.4), then

(iv) $\omega_{\tau, \Sigma}(B) \equiv \omega_{0, \Sigma}(B)$, that is, $\omega_{\tau, \Sigma}(B)$ is independent of $\tau \in \mathbb{R}$. 
Proof. (i) For any fixed $\tau \in \mathbb{R}$, then for any $t_{n} \in \mathbb{R}^{\tau}, t_{n} \rightarrow \infty, \sigma_{n} \in \Sigma$ and $x_{n} \in B$, by the definition of uniform (w.r.t. $\sigma \in \Sigma$ ) asymptotic compactness we know that $\left\{U_{\sigma_{n}}\left(t_{n}, \tau\right) x_{n}\right\}_{n=1}^{\infty}$ is precompact in $X$, without loss of generality, we assume that

$$
U_{\sigma_{n}}\left(t_{n}, \tau\right) x_{n} \rightarrow y
$$

Then by the definition of $\omega$-limit set we know that $y \in \omega_{\tau, \Sigma}(B)$, which implies that $\omega_{\tau, \Sigma}(B)$ is nonempty.

For any $y_{m} \in \omega_{\tau, \Sigma}(B), m=1,2, \cdots$, we will show that $\left\{y_{m}\right\}_{m=1}^{\infty}$ is precompact in $X$. By the definition, for each $m \in \mathbb{N}$, there exist $t_{m} \in \mathbb{R}^{\tau}, t_{m} \geqslant m, \sigma_{m} \in \Sigma$ and $x_{m} \in B$ such that

$$
\rho\left(U_{\sigma_{m}}\left(t_{m}, \tau\right) x_{m}, y_{m}\right) \leqslant \frac{1}{m},
$$

where $\rho(\cdot, \cdot)$ is the metric on $X$.

Therefore, by the assumption of uniform (w.r.t. $\sigma \in \Sigma$ ) asymptotic compactness again, we have $\left\{U_{\sigma_{m}}\left(t_{m}, \tau\right) x_{m}\right\}_{m=1}^{\infty}$ is precompact in $X$, and without loss of generality, we assume that $\left\{U_{\sigma_{m}}\left(t_{m}, \tau\right) x_{m}\right\}_{m=1}^{\infty}$ is a Cauchy sequence in $X$. Then, from

$$
\begin{aligned}
& \rho\left(y_{n}, y_{m}\right) \\
& \leqslant \rho\left(y_{n}, U_{\sigma_{n}}\left(t_{n}, \tau\right) x_{n}\right)+\rho\left(U_{\sigma_{n}}\left(t_{n}, \tau\right) x_{n}, U_{\sigma_{m}}\left(t_{m}, \tau\right) x_{m}\right)+\rho\left(U_{\sigma_{m}}\left(t_{m}, \tau\right) x_{m}, y_{m}\right) \\
& \leqslant \frac{1}{n}+\rho\left(U_{\sigma_{n}}\left(t_{n}, \tau\right) x_{n}, U_{\sigma_{m}}\left(t_{m}, \tau\right) x_{m}\right)+\frac{1}{m}
\end{aligned}
$$

we know that $\left\{y_{m}\right\}_{m=1}^{\infty}$ is also a Cauchy sequence in $X$. Moreover, from the definition we obviously have that $\omega_{\tau, \Sigma}(B)$ is closed in $X$.

Hence, $\omega_{\tau, \Sigma}(B)$ is compact in $X$.

(ii) If (ii) is not true, then there exist $\varepsilon_{0}>0, \sigma_{n} \in \Sigma, x_{n} \in B$ and $t_{n} \in \mathbb{R}^{\tau}$ with $t_{n} \geqslant n$, such that

$$
\operatorname{dist}\left(U_{\sigma_{n}}\left(t_{n}, \tau\right) x_{n}, \omega_{\tau, \Sigma}(B)\right) \geqslant \varepsilon_{0} \quad n=1,2, \cdots .
$$

However, the uniform (w.r.t. $\sigma \in \Sigma)$ asymptotic compactness implies that $\left\{U_{\sigma_{n}}\left(t_{n}, \tau\right) x_{n}\right\}_{n=1}^{\infty}$ is precompact in $X$, that is, $\left\{U_{\sigma_{n}}\left(t_{n}, \tau\right) x_{n}\right\}_{n=1}^{\infty}$ has a convergent subsequence which converges to some point of $\omega_{\tau, \Sigma}(B)$. This is a contradiction.

(iii) $\forall y \in \omega_{\tau, \Sigma}(B)$. Then there are $\sigma_{n} \in \Sigma, x_{n} \in B$ and $t_{n} \in \mathbb{R}^{\tau}$ with $t_{n} \rightarrow \infty$ such that $U_{\sigma_{n}}\left(t_{n}, \tau\right) x_{n} \rightarrow y$. From the assumption that $Y$ uniform attracts $B$, obviously, we have

$$
\operatorname{dist}\left(U_{\sigma_{n}}\left(t_{n}, \tau\right) x_{n}, Y\right) \rightarrow 0 \quad \text { as } n \rightarrow \infty .
$$

At the same time, the closeness of $Y$ implies $y \in Y$. Hence, $\omega_{\tau, \Sigma}(B) \subset Y$. 
(iv) For any fixed $\tau \in \mathbb{R}$ and $\sigma \in \Sigma$, from the translation identity (2.3) we know (e.g., see [29, 32]) that for any $\tau_{0} \in \mathbb{R}$ there is a $\sigma^{\prime} \in \Sigma$ such that

$$
U_{\sigma}(t, \tau)=U_{\sigma^{\prime}}\left(t-\tau+\tau_{0}, \tau_{0}\right), \quad \forall t \geqslant \tau .
$$

Combining with (2.4), we have that for any $t \geqslant \tau$,

$$
\bigcup_{\sigma \in \Sigma} \bigcup_{s \geqslant t} U_{\sigma}(s, \tau) B=\bigcup_{\sigma \in \Sigma} \bigcup_{s \geqslant t} U_{\sigma}(s-\tau, 0) B
$$

Therefore, we have

$$
\omega_{\tau, \Sigma}(B)=\bigcap_{t \geqslant \tau} \overline{\bigcup_{\sigma \in \Sigma} \bigcup_{s \geqslant t} U_{\sigma}(s, \tau) B}=\bigcap_{t \geqslant 0} \overline{\bigcup_{\sigma \in \Sigma} \bigcup_{s \geqslant t} U_{\sigma}(s, 0) B}=\omega_{0, \Sigma}(B) .
$$

Theorem 3.4. Let $X$ be a complete metric space, $\left\{U_{\sigma}(t, \tau)\right\}, \sigma \in \Sigma$ be a family of processes on $X$ and satisfies the translation identity (2.3)-(2.4). Then, $\left\{U_{\sigma}(t, \tau)\right\}, \sigma \in \Sigma$ has a compactly uniform (w.r.t. $\sigma \in \Sigma$ ) attractor $\mathscr{A}_{\Sigma}$ in $X$ and satisfies

$$
\mathscr{A}_{\Sigma}=\omega_{0, \Sigma}\left(B_{0}\right)=\omega_{\tau, \Sigma}\left(B_{0}\right)=\bigcup_{B \in \mathcal{B}(X)} \omega_{\tau, \Sigma}(B), \quad \forall \tau \in \mathbb{R},
$$

if and only if $\left\{U_{\sigma}(t, \tau)\right\}, \sigma \in \Sigma$

i) has a bounded uniformly (w.r.t. $\sigma \in \Sigma$ ) absorbing set $B_{0}$;

ii) is uniformly (w.r.t. $\sigma \in \Sigma$ ) asymptotically compact.

Proof. The necessity follows from the definition of uniform (w.r.t $\sigma \in \Sigma$ ) attractor and the compactness of $\mathscr{A}_{\Sigma}$.

Now we prove the sufficiency. For any fixed $\tau \in \mathbb{R}$ and any $B \in \mathcal{B}(X)$. We know that there is a $T=T(\tau, B)$ such that

$$
\bigcup_{\sigma \in \Sigma} \bigcup_{t \geqslant T} U_{\sigma}(t, \tau) B \subset B_{0} .
$$

Combining with the equivalent characterization, Proposition 3.2 of $\omega$-limit set and Lemma 3.3, we have

$$
\omega_{\tau, \Sigma}(B) \subset \omega_{\tau, \Sigma}\left(B_{0}\right)=\omega_{0, \Sigma}\left(B_{0}\right)
$$

and $\omega_{\tau, \Sigma}(B)$, of course, $\omega_{0, \Sigma}\left(B_{0}\right)$ uniform (w.r.t. $\sigma \in \Sigma$ ) attracts $B$.

Moreover, (3.2) implies that $\bigcup_{B \in \mathcal{B}(X)} \omega_{\tau, \Sigma}(B) \subset \omega_{0, \Sigma}\left(B_{0}\right)$, and from $B_{0} \in \mathcal{B}(X)$ we obtain $\bigcup_{B \in \mathcal{B}(X)} \omega_{\tau, \Sigma}(B)=\omega_{0, \Sigma}\left(B_{0}\right)$.

The minimality and closeness follows immediately from (iii) of Lemma 3.3, and the compactness follows from (i) of Lemma 3.3 . 


\subsection{Structure of the uniform attractor}

We describe the structure of the uniform attractor by means of its kernel sections.

Hereafter, we assume that $X$ is a Banach space with norm $\|\cdot\|_{X}$ and $\Sigma$ is a complete metric space with metric $d(\cdot, \cdot)$.

Let $\{U(t, \tau) \mid t \geqslant \tau, \tau \in \mathbb{R}\}=\{U(t, \tau)\}$ be a process acting in a Banach space $X$, and let $\mathcal{K}$ be the kernel of the process $\{U(t, \tau)\}$. We recall (e.g., see [11]) that the kernel $\mathcal{K}$ consists of all bounded complete trajectories of the process, i.e.,

$$
\mathcal{K}=\left\{u(\cdot) \mid\|u(t)\| X \leqslant C_{u}, U(t, \tau) u(\tau)=u(t), \forall t \geqslant \tau, \tau \in \mathbb{R}\right\}
$$

and $\mathcal{K}(s)$ denotes the kernel section at a time moment $s \in \mathbb{R}$ :

$$
\mathcal{K}(s)=\{u(s) \mid u(\cdot) \in \mathcal{K}\}, \quad \mathcal{K}(s) \subset X .
$$

As mentioned in [11], since the invariance of the global attractor of a semigroup is replaced by the minimality in the definition of the uniform attractor of a family of processes, the existence of uniform attractor dose not need any continuity for the processes. However, in order to obtain the structure of the uniform attractor, the continuity maybe necessary to some extend.

\subsubsection{Norm-to-weak continuous processes}

In [9, 11], in order to obtain the structure of the uniform attractor, the authors assume that the family of processes $\left\{U_{\sigma}(t, \tau)\right\}, \sigma \in \Sigma$ is $(X \times \Sigma, X)$-continuous, see Theorem 5.1 in Chapter IV of [1].

Now, as noticed in [40, in order to obtain the invariance of the global attractor of a semigroup for an autonomous system, we only need the norm-to-weak continuity. In this part, we will generalize these results to non-autonomous systems.

Definition 3.5. A family of processes $\left\{U_{\sigma}(t, \tau)\right\}, \sigma \in \Sigma$ is said to be norm-to-weak continuous, if for any fixed $t$ and $\tau \in \mathbb{R}$ with $t \geqslant \tau$, for any $\left\{x_{n}\right\} \subset X$ and $\left\{\sigma_{n}\right\} \subset \Sigma$, we have

$$
\left.\begin{array}{r}
x_{n} \stackrel{\|\cdot\|_{X}}{\rightarrow} x \\
\sigma_{n} \stackrel{d}{\rightarrow} \sigma
\end{array}\right\} \Rightarrow U_{\sigma_{n}}(t, \tau) x_{n} \rightarrow U_{\sigma}(t, \tau) x \quad \text { weakly in } X .
$$

For convenience, we also use the following notations:

Definition 3.6. A semigroup $\{S(t)\}_{t \geqslant 0}: X \times \Sigma \rightarrow X \times \Sigma$ is to be called skew productively norm-to-weak continuous, if for any fixed $t \geqslant 0$, for any $\left\{x_{n}\right\} \subset X$ and $\left\{\sigma_{n}\right\} \subset \Sigma$, 
we have

$$
\begin{aligned}
& \Pi_{1} S(t)\left(x_{n}, \sigma_{n}\right) \stackrel{\text { weak }}{\rightarrow} \Pi_{1} S(t)(x, \sigma), \\
& \Pi_{2} S(t)\left(x_{n}, \sigma_{n}\right) \stackrel{d}{\rightarrow} \Pi_{2} S(t)(x, \sigma)
\end{aligned}
$$

provided that $x_{n} \stackrel{\|\cdot\|_{X}}{\rightarrow} x$ and $\sigma_{n} \stackrel{d}{\rightarrow} \sigma$, where $\Pi_{1}$ and $\Pi_{2}$ are the canonical projector from $X \times \Sigma$ to $X$ and $\Sigma$, respectively. Denoted such continuity by

$$
S(t)\left(x_{n}, \sigma_{n}\right) \stackrel{s-w}{\longrightarrow} S(t)(x, \sigma) .
$$

Following from the definition of skew productively norm-to-weak continuous semigroup, we have the following result.

Proposition 3.7. Let $\left\{U_{\sigma}(t, \tau)\right\}, \sigma \in \Sigma$ be a family norm-to-weak continuous processes in $X$, and the translation semigroup $\{T(t)\}_{t \geqslant 0}$ is continuous (w.r.t. the metric $\left.d(\cdot, \cdot)\right)$ in $\Sigma$. Then, the semigroup $\{S(t)\}_{t \geqslant 0}$ corresponding to $\left\{U_{\sigma}(t, \tau)\right\}, \sigma \in \Sigma$, defined by (2.5) and acting on $X \times \Sigma$, is skew productively norm-to-weak continuous.

\subsubsection{Kernel sections of the uniform attractor}

Theorem 3.8. Let $X$ be a Banach space and $\Sigma$ be a compact metric space. Assume that a family of processes $\left\{U_{\sigma}(t, \tau)\right\}, \sigma \in \Sigma$ satisfies the translation identity (2.3)-(2.4), as well as the following conditions:

(i) The translation semigroup $\{T(t)\}_{t \geqslant 0}$ is continuous on $\Sigma$;

(ii) $\left\{U_{\sigma}(t, \tau)\right\}, \sigma \in \Sigma$ is norm-to-weak continuous on $X$;

(iii) $\left\{U_{\sigma}(t, \tau)\right\}, \sigma \in \Sigma$ has a bounded uniformly (w.r.t. $\sigma \in \Sigma$ ) absorbing set $B_{0}$ in $X$;

(iv) $\left\{U_{\sigma}(t, \tau)\right\}, \sigma \in \Sigma$ is uniform (w.r.t. $\sigma \in \Sigma$ ) asymptotically compact in $X$.

Then, $\left\{U_{\sigma}(t, \tau)\right\}, \sigma \in \Sigma$ has a uniform (w.r.t. $\sigma \in \Sigma$ ) attractor $\mathscr{A}_{\Sigma}$ satisfying

$$
\mathscr{A}_{\Sigma}=\omega_{0, \Sigma}\left(B_{0}\right)=\bigcup_{\sigma \in \Sigma} \mathcal{K}_{\sigma}(s), \quad \forall s \in \mathbb{R},
$$

where $\mathcal{K}_{\sigma}(s)$ is the section at $t=s$ of the kernel $\mathcal{K}_{\sigma}$ of the process $\left\{U_{\sigma}(t, \tau)\right\}$ with symbol $\sigma$.

Proof. From the assumptions (iii), (iv) and Theorem 3.4, we know that the family of processes $\left\{U_{\sigma}(t, \tau)\right\}, \sigma \in \Sigma$ has a compactly uniform (w.r.t. $\sigma \in \Sigma$ ) attractor $\mathscr{A}_{\Sigma}$ which satisfies

$$
\mathscr{A}_{\Sigma}=\omega_{0, \Sigma}\left(B_{0}\right)
$$


In order to prove the structure (3.3), we will construct skew product flow on $X \times \Sigma$. We will complete the proof by three steps.

Step 1. Constructing skew product flow $\{S(t)\}_{t \geqslant 0}$ on $X \times \Sigma$ :

$$
S(t)(u, \sigma)=\left(U_{\sigma}(t, 0) u, T(t) \sigma\right), \quad t \geqslant 0, \quad(u, \sigma) \in X \times \Sigma .
$$

Then follows from (2.3) and Proposition 3.7 we have that $\{S(t)\}_{t \geqslant 0}$ forms a skew productively norm-to-weak continuous semigroup on $X \times \Sigma$.

Define the $\omega$-limit set of $\{S(t)\}_{t \geqslant 0}$ by

$$
\mathscr{A}=\omega\left(B_{0} \times \Sigma\right)=\bigcap_{t \geqslant 0} \overline{\bigcup_{s \geqslant t} S(s)\left(B_{0} \times \Sigma\right)},
$$

where $\bar{A}$ denotes the closure of $A$ in $X \times \Sigma$. Then we have the following equivalent characterization:

$$
\begin{gathered}
(x, \sigma) \in \mathscr{A} \text { if and only if there exist } x_{n} \in B_{0}, \sigma_{n} \in \Sigma \text { and } t_{n} \geqslant 0 \text { with } t_{n} \rightarrow \infty, \\
\text { such that } S\left(t_{n}\right)\left(x_{n}, \sigma_{n}\right) \rightarrow(x, \sigma) \text { as } n \rightarrow \infty \text { in } X \times \Sigma .
\end{gathered}
$$

Step 2. From the assumptions that $\Sigma$ is a compact metric space and conditions (iii) and (iv) we have that $\mathscr{A}$ is nonempty, compact in $X \times \Sigma$, and attracts every bounded subset of $X \times \Sigma$ under the topology of $X \times \Sigma$. In order to show that $\mathscr{A}$ is a global attractor of $\{S(t)\}_{t \geqslant 0}$ in $X \times \Sigma$, we need to show the invariance of $\mathscr{A}$, that is, we need to prove

$$
S(t) \mathscr{A}=\mathscr{A} \quad \forall t \geqslant 0 .
$$

For any $(x, \sigma) \in \mathscr{A}$ and $t \geqslant 0$. From (3.5) we know that there exist $x_{n} \in B_{0}, \sigma_{n} \in \Sigma$ and $t_{n} \geqslant 0, t_{n} \rightarrow \infty$ such that $S\left(t_{n}\right)\left(x_{n}, \sigma_{n}\right) \rightarrow(x, \sigma)$. Since $t_{n} \rightarrow \infty$, without loss of generality, we assume that $t_{n} \geqslant t$ for each $n$ (at most by passing subsequence). Then from (3.4) we have

$$
S\left(t_{n}-t\right)\left(x_{n}, \sigma_{n}\right)=\left(U_{\sigma_{n}}\left(t_{n}-t, 0\right) x_{n}, T\left(t_{n}-t\right) \sigma_{n}\right) .
$$

Noticing that $\left\{x_{n}\right\} \subset B_{0}$ and $t_{n}-t \rightarrow \infty$, then by the assumption (iv), we know that there is a convergent subsequence of $\left\{U_{\sigma_{n}}\left(t_{n}-t, 0\right) x_{n}\right\}$, without loss of generality, we also assume that $U_{\sigma_{n}}\left(t_{n}-t, 0\right) x_{n} \rightarrow y$ for some $y \in X$ as $n \rightarrow \infty$. At the same time, by the assumption that $\Sigma$ is a compact metric space, without loss of generality, we can also assume that $T\left(t_{n}-t\right) \sigma_{n} \rightarrow \sigma_{0}$ for some $\sigma_{0} \in \Sigma$ as $n \rightarrow \infty$. Consequently, $S\left(t_{n}-t\right)\left(x_{n}, \sigma_{n}\right) \rightarrow\left(y, \sigma_{0}\right)$ as $n \rightarrow \infty$. Then from the definition we have that $\left(y, \sigma_{0}\right) \in \mathscr{A}$. Moreover, from the skew productively norm-to-weak continuity of $\{S(t)\}_{t \geqslant 0}$, we have

$$
(x, \sigma) \leftarrow S\left(t_{n}\right)\left(x_{n}, \sigma_{n}\right)=S(t) S\left(t_{n}-t\right)\left(x_{n}, \sigma_{n}\right) \stackrel{s-w}{\longrightarrow} S(t)\left(y, \sigma_{0}\right) .
$$


Following the uniqueness of limits, we have $(x, \sigma)=S(t)\left(y, \sigma_{0}\right)$, i.e., $\mathscr{A} \subset S(t) \mathscr{A}$.

Similarly, we can prove $S(t) \mathscr{A} \subset \mathscr{A}$. Therefore, $\mathscr{A}$ is the global attractor of $\{S(t)\}_{t \geqslant 0}$ and we have

$$
\mathscr{A}=\left\{\gamma(0) \mid \gamma(\cdot) \text { is a bounded complete trajectory of }\{S(t)\}_{t \geqslant 0}\right\}
$$

Step 3. Based on Step 1 and Step 2, similar to what done in Chepyzhov \& Vishik [11], Theorem 5.1, Chapter IV], we can prove that $\mathscr{A}_{\Sigma}=\Pi_{1} \mathscr{A}=\bigcup_{\sigma \in \Sigma} \mathcal{K}_{\sigma}(s)$ and $\Pi_{2} \mathscr{A}=\Sigma$.

In practical applications, $\Sigma$ would be the completion of a dense subset $\Sigma_{0}(\subset \Sigma)$ with respect to some metric $d(\cdot, \cdot)$, and maybe different for different metrics. For example, $\Sigma_{0}=\left\{g_{0}(s+h, x) \mid h \in \mathbb{R}\right\}$ for some function $g_{0}(s, x)$ belong to a special function space, and $\Sigma$ can be chosen according to our concrete problem.

If the family of processes $\left\{U_{\sigma}(t, \tau)\right\}, \sigma \in \Sigma_{0}$ satisfies also the translation identity (2.3)(2.4), then, Chepyzhov \& Vishik proved in [9, 11] that the uniform (w.r.t $\tau \in \mathbb{R}$ ) attractor of a process $\left\{U_{\sigma_{0}}(t, \tau)\right\}, \tau \in \mathbb{R}$ coincides with the uniform (w.r.t. $\sigma \in \Sigma_{0}$ ) attractor for the family of processes $\left\{U_{\sigma}(t, \tau)\right\}, \sigma \in \Sigma_{0}$.

Similar to [1], 29], the following results give a method to obtain the structure of the uniform (w.r.t $\tau \in \mathbb{R}$ ) attractor of a process $\left\{U_{\sigma_{0}}(t, \tau)\right\}, \tau \in \mathbb{R}$ via the structure of the uniform (w.r.t. $\sigma \in \Sigma_{0}$ ) attractor for the family of processes $\left\{U_{\sigma}(t, \tau)\right\}, \sigma \in \Sigma_{0}$.

Since our processes are norm-to-weak continuity, we first give a simple lemma about metrizable. We recall (e.g., see Diestel[17], p. 18) that a set $F \subset X^{*}$ is called total if $f(x)=0$ for each $f \in F$ implies $x=0$.

Lemma 3.9. If $K$ is a (relatively) weakly compact subset in a Banach space $X$ and $K$ is countable, then $\bar{K}^{\text {weak }}$ is metrizable, where $\bar{K}^{\text {weak }}$ means the weak closure of $K$ in $X$.

Proof. Denote $Y=\overline{\operatorname{span}\{K\}}$.

From the convexity of $\operatorname{span}\{K\}$ we know $Y$ is weakly closed in $X$. Therefore, $K=$ $K \cap Y$ is (relatively) weakly compact in the separable Banach space $Y$. Since the dual of a separable Banach space contains a countable total set, we know that $\bar{K}^{\text {weak }}$ is metrizable in $Y$, and from that $Y$ is a closed subspace of $X$ we get $\bar{K}^{\text {weak }}$ is metrizable in $X$.

Theorem 3.10. Let $\Sigma_{0}$ be a parameter set, $\Sigma$ is a completion of $\Sigma_{0}$ with respect to some metric $d(\cdot, \cdot)$, and the translation semigroup $\{T(t)\}_{t \geqslant 0}$ satisfies also the translation identity (2.3)-(2.4) on $\Sigma_{0}$. Furthermore, assume that the family of processes $\left\{U_{\sigma}(t, \tau)\right\}, \sigma \in$ $\Sigma$ satisfies all of the assumptions in Theorem [3.8. Then, both families of processes 
$\left\{U_{\sigma}(t, \tau)\right\}, \sigma \in \Sigma$ and $\sigma \in \Sigma_{0}$ have compactly uniform (w.r.t. $\sigma \in \Sigma$ and $\sigma \in \Sigma_{0}$ respectively) attractors $\mathscr{A}_{\Sigma}$ and $\mathscr{A}_{\Sigma_{0}}$ respectively, moreover,

$$
\mathscr{A}_{\Sigma_{0}}=\mathscr{A}_{\Sigma}=\omega_{0, \Sigma}\left(B_{0}\right)=\bigcup_{\sigma \in \Sigma} \mathcal{K}_{\sigma}(s), \quad \forall s \in \mathbb{R}
$$

Proof. The existence is a immediate consequence of Theorem 3.8, and obviously, we have

$$
\mathscr{A}_{\Sigma_{0}} \subset \mathscr{A}_{\Sigma}=\omega_{0, \Sigma}\left(B_{0}\right)=\bigcup_{\sigma \in \Sigma} \mathcal{K}_{\sigma}(s), \quad \forall s \in \mathbb{R}
$$

Now we prove $\omega_{0, \Sigma_{0}}\left(B_{0}\right)=\omega_{0, \Sigma}\left(B_{0}\right)$. For any $y \in \omega_{0, \Sigma}\left(B_{0}\right)$, from Proposition 3.2 we know that there exist $x_{n} \in B_{0}, t_{n} \rightarrow \infty$ and $\sigma_{n} \in \Sigma$ such that

$$
U_{\sigma_{n}}\left(t_{n}, 0\right) x_{n} \rightarrow y \quad \text { as } n \rightarrow \infty
$$

On the other hand, from the assumption that $\Sigma$ is the completion of $\Sigma_{0}$ we know that there exists $\left\{\sigma_{m}^{(n)}\right\} \subset \Sigma_{0}$ satisfies $\sigma_{m}^{(n)} \stackrel{d}{\rightarrow} \sigma_{n}$ as $m \rightarrow \infty$ for each $n \in \mathbb{N}$. Therefore, due to the norm-to-weak continuity of the family of processes $\left\{U_{\sigma}(t, \tau)\right\}, \sigma \in \Sigma$, we have

$$
U_{\sigma_{m}^{(n)}}\left(t_{n}, 0\right) x_{n} \rightarrow U_{\sigma_{n}}\left(t_{n}, 0\right) x_{n} \quad \text { as } m \rightarrow \infty
$$

for each $n \in \mathbb{N}$. Denote $K=\left\{U_{\sigma_{m}^{(n)}}\left(t_{n}, 0\right) x_{n} \mid n, m \in \mathbb{N}\right\}$, then $K$ is countable and thanks to the condition (iv) of Theorem 3.8 we know $K$ is also relatively weakly compact in $X$. Consequently, from Lemma 3.9 we have that $\bar{K}^{\text {weak }}$ is metrizable.

Hence, combining (3.6) and (3.7), we can obtain that there exist $\sigma_{n}^{\prime} \in \Sigma_{0}$ for each $n \in \mathbb{N}$ such that

$$
U_{\sigma_{n}^{\prime}}\left(t_{n}, 0\right) x_{n} \rightarrow y \quad \text { in } X \text { as } n \rightarrow \infty \text {. }
$$

Then noticing the uniform asymptotic compactness again and the uniqueness of limits, we have $y \in \omega_{0, \Sigma_{0}}\left(B_{0}\right)$.

\section{A criterion for verifying the uniform asymptotic compact- ness}

In this section, we present a technical method to verify the uniform asymptotic compactness (given in Definition 3.1) for the family of processes generated by non-autonomous hyperbolic type of evolutionary equations. This criterion is partially motivated by the methods in [12, 13, 14, 24, 37] for autonomous systems. Here, the following results and proof are similar to that in [37] for autonomous cases. 
Definition 4.1. Let $X$ be a Banach space and $B$ be a bounded subset of $X, \Sigma$ be a symbol (or parameter) space. We call a function $\phi(\cdot, \cdot ; \cdot, \cdot)$, defined on $(X \times X) \times(\Sigma \times \Sigma)$, to be a contractive function on $B \times B$ if for any sequence $\left\{x_{n}\right\}_{n=1}^{\infty} \subset B$ and any $\left\{\sigma_{n}\right\} \subset \Sigma$, there is a subsequence $\left\{x_{n_{k}}\right\}_{k=1}^{\infty} \subset\left\{x_{n}\right\}_{n=1}^{\infty}$ and $\left\{\sigma_{n_{k}}\right\}_{k=1}^{\infty} \subset\left\{\sigma_{n}\right\}_{n=1}^{\infty}$ such that

$$
\lim _{k \rightarrow \infty} \lim _{l \rightarrow \infty} \phi\left(x_{n_{k}}, x_{n_{l}} ; \sigma_{n_{k}}, \sigma_{n_{l}}\right)=0 .
$$

We denote the set of all contractive functions on $B \times B$ by $\mathfrak{C}(B, \Sigma)$.

Theorem 4.2. Let $\left\{U_{\sigma}(t, \tau)\right\}, \sigma \in \Sigma$ be a family of processes satisfies the translation identity (2.3)-(2.4) on Banach space $X$ and has a bounded uniformly (w.r.t. $\sigma \in \Sigma$ ) absorbing set $B_{0} \subset X$. Moreover, assume that for any $\varepsilon>0$ there exist $T=T\left(B_{0}, \varepsilon\right)$ and $\phi_{T} \in \mathfrak{C}\left(B_{0}, \Sigma\right)$ such that

$$
\left\|U_{\sigma_{1}}(T, 0) x-U_{\sigma_{2}}(T, 0) y\right\| \leqslant \varepsilon+\phi_{T}\left(x, y ; \sigma_{1}, \sigma_{2}\right), \quad \forall x, y \in B_{0}, \forall \sigma_{1}, \sigma_{2} \in \Sigma .
$$

Then $\left\{U_{\sigma}(t, \tau)\right\}, \sigma \in \Sigma$ is uniformly (w.r.t. $\sigma \in \Sigma$ ) asymptotically compact in $X$.

Proof. For any fixed $\tau \in \mathbb{R}$, let $\left\{x_{n}\right\}_{n=1}^{\infty}$ be a bounded sequence of $X, \sigma_{n} \in \Sigma$ and $t_{n} \geqslant \tau$ satisfy $t_{n} \rightarrow \infty$ as $n \rightarrow \infty$. We need to show that

$$
\left\{U_{\sigma_{n}}\left(t_{n}, \tau\right) x_{n}\right\}_{n=1}^{\infty} \text { is precompact in } X \text {. }
$$

Thanks to the translation identity (2.3)-(2.4), we know that for any fixed $\tau \in \mathbb{R}$ and $\sigma \in \Sigma$ we can find $\sigma^{\prime} \in \Sigma$ such that

$$
U_{\sigma^{\prime}}(t+\tau, \tau) x=U_{\sigma}(t, 0) x, \quad \text { for all } t \geqslant 0 \text { and } x \in X
$$

Therefore, we only need to show that $\left\{U_{\sigma_{n}}\left(t_{n}, 0\right) x_{n}\right\}_{n=1}^{\infty}$ is precompact in $X$.

In the following, we will prove that $\left\{U_{\sigma_{n}}\left(t_{n}, 0\right) x_{n}\right\}_{n=1}^{\infty}$ has a Cauchy subsequence via a diagonal method.

Taking $\varepsilon_{m}>0$ with $\varepsilon_{m} \rightarrow 0$ as $m \rightarrow \infty$.

At first, for $\varepsilon_{1}$, by the assumptions, there exist $T_{1}=T_{1}\left(\varepsilon_{1}\right)$ and $\phi_{1} \in \mathfrak{C}\left(B_{0}, \Sigma\right)$ such that

$\left\|U_{\sigma_{1}}\left(T_{1}, 0\right) x-U_{\sigma_{2}}\left(T_{1}, 0\right) y\right\| \leqslant \varepsilon_{1}+\phi_{1}\left(x, y ; \sigma_{1}, \sigma_{2}\right)$ for all $x, y \in B_{0}$ and $\sigma_{1}, \sigma_{2} \in \Sigma$.

Since $t_{n} \rightarrow \infty$, for such fixed $T_{1}$, without loss of generality, we assume that $t_{n} \gg T_{1}$ is so large that $U_{\sigma_{n}}\left(t_{n}-T_{1}, 0\right) x_{n} \in B_{0}$ for each $n \in \mathbb{N}$.

Similar to (4.1), for each $n \in \mathbb{N}$, there is a $\sigma_{n}^{\prime} \in \Sigma$ such that

$$
U_{\sigma_{n}^{\prime}}\left(T_{1}, 0\right)=U_{\sigma_{n}}\left(t_{n}, t_{n}-T_{1}\right)
$$


Let $y_{n}=U_{\sigma_{n}}\left(t_{n}-T_{1}, 0\right) x_{n}$, then from (4.2) and (4.3) we have

$$
\begin{aligned}
\| U_{\sigma_{n}} & \left(t_{n}, 0\right) x_{n}-U_{\sigma_{m}}\left(t_{m}, 0\right) x_{m} \| \\
& =\left\|U_{\sigma_{n}}\left(t_{n}, t_{n}-T_{1}\right) U_{\sigma_{n}}\left(t_{n}-T_{1}, 0\right) x_{n}-U_{\sigma_{m}}\left(t_{m}, t_{m}-T_{1}\right) U_{\sigma_{n}}\left(t_{m}-T_{1}, 0\right) x_{m}\right\| \\
& =\left\|U_{\sigma_{n}}\left(t_{n}, t_{n}-T_{1}\right) y_{n}-U_{\sigma_{m}}\left(t_{m}, t_{m}-T_{1}\right) y_{m}\right\| \\
& =\left\|U_{\sigma_{n}^{\prime}}\left(T_{1}, 0\right) y_{n}-U_{\sigma_{m}^{\prime}}\left(T_{1}, 0\right) y_{m}\right\| \\
& \leqslant \varepsilon_{1}+\phi_{1}\left(y_{n}, y_{m} ; \sigma_{n}^{\prime}, \sigma_{m}^{\prime}\right) .
\end{aligned}
$$

Due to the definition of $\mathfrak{C}\left(B_{0}, \Sigma\right)$ and $\phi_{1} \in \mathfrak{C}\left(B_{0}, \Sigma\right)$, we know that $\left\{y_{n}\right\}_{n=1}^{\infty}$ has a subsequence $\left\{y_{n_{k}}^{(1)}\right\}_{k=1}^{\infty}$ and $\left\{\sigma_{n}^{\prime}\right\}_{n=1}^{\infty}$ has a subsequence $\left\{\sigma_{n_{k}}^{\prime(1)}\right\}_{k=1}^{\infty}$ such that

$$
\lim _{k \rightarrow \infty} \lim _{l \rightarrow \infty} \phi_{1}\left(y_{n_{k}}^{(1)}, y_{n_{l}}^{(1)} ; \sigma_{n_{k}}^{\prime(1)}, \sigma_{n_{l}}^{\prime(1)}\right) \leqslant \frac{\varepsilon_{1}}{2} .
$$

And similar to the autonomous cases, e.g., see [24, 37], we have

$$
\begin{gathered}
\limsup _{k \rightarrow \infty}\left\|U_{\sigma_{n}(1)}\left(t_{n_{k+p}}^{(1)}, 0\right) x_{n_{k+p}}^{(1)}-U_{\sigma_{n_{k}}^{(1)}}\left(t_{n_{k}}^{(1)}, 0\right) x_{n_{k}}^{(1)}\right\| \\
\leqslant \lim _{k \rightarrow \infty} \sup _{p \in \mathbb{N}} \limsup _{l \rightarrow \infty}\left\|U_{\sigma_{n_{k+p}}^{(1)}}\left(t_{n_{k+p}}^{(1)}, 0\right) x_{n_{k+p}}^{(1)}-U_{\sigma_{n_{l}}^{(1)}}\left(t_{n_{l}}^{(1)}, 0\right) x_{n_{l}}^{(1)}\right\| \\
\quad+\limsup _{k \rightarrow \infty} \limsup _{l \rightarrow \infty}\left\|U_{\sigma_{n_{k}}^{(1)}}\left(t_{n_{k}}^{(1)}, 0\right) x_{n_{k}}^{(1)}-U_{\sigma_{n_{l}}^{(1)}}\left(t_{n_{l}}^{(1)}, 0\right) x_{n_{l}}^{(1)}\right\| \\
\leqslant \varepsilon_{1}+\lim _{k \rightarrow \infty} \sup _{p \in \mathbb{N}} \lim _{l \rightarrow \infty} \phi_{1}\left(y_{n_{k+p}}^{(1)}, y_{n_{l}}^{(1)} ; \sigma_{n_{k+p}}^{(1)}, \sigma_{n_{l}}^{(1)}\right) \\
\quad+\varepsilon_{1}+\lim _{k \rightarrow \infty} \lim _{l \rightarrow \infty} \phi_{1}\left(y_{n_{k}}^{(1)}, y_{n_{l}}^{(1)} ; \sigma_{n_{k}}^{(1)}, \sigma_{n_{l}}^{(1)}\right)
\end{gathered}
$$

which, combining with (4.4) and (4.5), implies that

$$
\lim _{k \rightarrow \infty} \sup _{p \in \mathbb{N}}\left\|U_{\sigma_{n_{k+p}}^{(1)}}\left(t_{n_{k+p}}^{(1)}, 0\right) x_{n_{k+p}}^{(1)}-U_{\sigma_{n_{k}}^{(1)}}\left(t_{n_{k}}^{(1)}, 0\right) x_{n_{k}}^{(1)}\right\| \leqslant 4 \varepsilon_{1} .
$$

Therefore, there is a $K_{1}$ such that

$$
\left\|U_{\sigma_{n_{k}}^{(1)}}\left(t_{n_{k}}^{(1)}, 0\right) x_{n_{k}}^{(1)}-U_{\sigma_{n_{l}}^{(1)}}\left(t_{n_{l}}^{(1)}, 0\right) x_{n_{l}}^{(1)}\right\| \leqslant 5 \varepsilon_{1} \quad \forall k, l \geqslant K_{1} .
$$

By induction, we obtain that, for each $m \geqslant 1$, there is a subsequence $\left\{U_{\sigma_{n_{k}}^{(m+1)}}\left(t_{n_{k}}^{(m+1)}, 0\right) x_{n_{k}}^{(m+1)}\right\}_{k=1}^{\infty}$ of $\left\{U_{\sigma_{n_{k}}^{(m)}}\left(t_{n_{k}}^{(m)}, 0\right) x_{n_{k}}^{(m)}\right\}_{k=1}^{\infty}$ and certain $K_{m+1}$ such that

$$
\left\|U_{\sigma_{n_{k}}^{(m+1)}}\left(t_{n_{k}}^{(m+1)}, 0\right) x_{n_{k}}^{(m+1)}-U_{\sigma_{n_{l}}^{(m+1)}}\left(t_{n_{l}}^{(m+1)}, 0\right) x_{n_{l}}^{(m+1)}\right\| \leqslant 5 \varepsilon_{m+1} \quad \forall k, l \geqslant K_{m+1} .
$$

Now, we consider the diagonal subsequence $\left\{U_{\sigma_{n_{k}}^{(k)}}\left(t_{n_{k}}^{(k)}, 0\right) x_{n_{k}}^{(k)}\right\}_{k=1}^{\infty}$. Since for each $m \in \mathbb{N},\left\{U_{\sigma_{n_{k}}^{(k)}}\left(t_{n_{k}}^{(k)}, 0\right) x_{n_{k}}^{(k)}\right\}_{k=m}^{\infty}$ is a subsequence of $\left\{U_{\sigma_{n_{k}}^{(m)}}\left(t_{n_{k}}^{(m)}, 0\right) x_{n_{k}}^{(m)}\right\}_{k=1}^{\infty}$, then,

$$
\left\|U_{\sigma_{n_{k}}^{(k)}}\left(t_{n_{k}}^{(k)}, 0\right) x_{n_{k}}^{(k)}-U_{\sigma_{n_{l}}^{(l)}}\left(t_{n_{l}}^{(l)}, 0\right) x_{n_{l}}^{(l)}\right\| \leqslant 5 \varepsilon_{m} \quad \forall k, l \geqslant \max \left\{m, K_{m}\right\},
$$

which, combining with $\varepsilon_{m} \rightarrow 0$ as $m \rightarrow \infty$, implies that $\left\{U_{\sigma_{n_{k}}^{(k)}}\left(t_{n_{k}}^{(k)}, 0\right) x_{n_{k}}^{(k)}\right\}_{k=m}^{\infty}$ is a Cauchy sequence in $X$. This shows that $\left\{U_{\sigma_{n}}\left(t_{n}, 0\right) x_{n}\right\}_{n=1}^{\infty}$ is precompact in $X$. 


\section{Application to wave equation}

\subsection{Mathematical setting}

Similar to the autonomous cases (e.g., see [13]), applying the Galerkin approximation method, we have the following existence and uniqueness results (e.g., see [22, 27]), and the time-dependent terms make no essential complications.

Theorem 5.1. Let $\Omega$ be a bounded domain of $\mathbb{R}^{3}$ with smooth boundary, $h$ and $f$ satisfy (1.4)-(1.8), and $g \in L^{\infty}\left(\mathbb{R} ; L^{2}(\Omega)\right)$. Then the non-autonomous initial-boundary value problem (1.1) -(1.3) has an unique solution $u(t)$ satisfying $\left(u(t), u_{t}(t)\right) \in \mathcal{C}\left(\mathbb{R}^{\tau} ; H_{0}^{1}(\Omega) \times\right.$ $\left.L^{2}(\Omega)\right)$ and $\partial_{t t} u(t) \in L_{l o c}^{2}\left(\mathbb{R}^{\tau} ; H^{-1}(\Omega)\right)$ for any initial data $\left(u^{0 \tau}, u^{1 \tau}\right) \in H_{0}^{1}(\Omega) \times L^{2}(\Omega)$.

We use the notations as in Chepyzhov \& Vishik [1]: Let $y(t)=\left(u(t), u_{t}(t)\right), y_{\tau}=$ $\left(u^{0 \tau}, u^{1 \tau}\right)$ and $X=H_{0}^{1}(\Omega) \times L^{2}(\Omega)$ with finite energy norm

$$
\|y\|_{X}=\left\{\|\nabla u\|^{2}+\left|u_{t}\right|^{2}\right\}^{\frac{1}{2}} .
$$

Let $A_{\sigma(t)}(u, v)=(v, \Delta u-f(u)-h(v)+\sigma(t))$. Then the non-autonomous system (1.1)-(1.3) can be rewritten in the operator form

$$
\partial_{t} y=A_{\sigma(t)}(y),\left.\quad y\right|_{t=\tau}=y_{\tau}
$$

where $\sigma(s)=g(x, s)$ is symbol of equation (5.1).

We now define the symbol space for (5.1). Taking a fixed symbol $\sigma_{0}(s)=g_{0}(x, s)$, $g_{0} \in L^{\infty}\left(\mathbb{R} ; L^{2}(\Omega)\right) \cap W_{b}^{1, r}\left(\mathbb{R} ; L^{r}(\Omega)\right)$. Set

$$
\Sigma_{0}=\left\{g_{0}(x, t+h) \mid h \in \mathbb{R}\right\}
$$

and

$$
\Sigma \text { be the } * \text {-weakly closure of } \Sigma_{0} \text { in } L^{\infty}\left(\mathbb{R} ; L^{2}(\Omega)\right) \cap W_{b}^{1, r}\left(\mathbb{R} ; L^{r}(\Omega)\right) \text {. }
$$

Then we have the following simple properties.

\section{Proposition 5.2.}

(i) $\Sigma$ is bounded in $L^{\infty}\left(\mathbb{R} ; L^{2}(\Omega)\right) \cap W_{b}^{1, r}\left(\mathbb{R} ; L^{r}(\Omega)\right)$, and for any $\sigma \in \Sigma$, the following estimate holds

$$
\|\sigma\|_{L^{\infty}\left(\mathbb{R} ; L^{2}(\Omega)\right) \cap W_{b}^{1, r}\left(\mathbb{R} ; L^{r}(\Omega)\right)} \leqslant\left\|g_{0}\right\|_{L^{\infty}\left(\mathbb{R} ; L^{2}(\Omega)\right) \cap W_{b}^{1, r}\left(\mathbb{R} ; L^{r}(\Omega)\right)}
$$

(ii) The translation semigroup $\{T(h) \mid h \geqslant 0\}$ acting on $\Sigma$ is invariant in $\Sigma$, that is

$$
T(h) \Sigma=\Sigma \quad \text { for all } h \in \mathbb{R}^{+} .
$$


Thus, from Theorem [5.1, we know that (1.1)-(1.3) is well posed for all $\sigma(s) \in \Sigma$ and generates a family of processes $\left\{U_{\sigma}(t, \tau)\right\}, \sigma \in \Sigma$ given by the formula $U_{\sigma}(t, \tau) y^{\tau}=y(t)$, where $y(t)$ is the solution of (1.1)-(1.8), and $\left\{U_{\sigma}(t, \tau)\right\}, \sigma \in \Sigma$ satisfies (2.1)-(2.2). At the same time, by the unique solvability, we know $\left\{U_{\sigma}(t, \tau)\right\}, \sigma \in \Sigma$ satisfies the translation identity (2.3).

In what follows, we denote by $\left\{U_{\sigma}(t, \tau)\right\}, \sigma \in \Sigma$ the family of processes generated by (5.1) $-(5.3)$.

\subsection{Bounded uniformly (w.r.t. $\sigma \in \Sigma$ ) absorbing set}

We begin with the following result on the existence of bounded uniformly (w.r.t. $\sigma \in \Sigma$ ) absorbing set. Its proof is essentially established in Haraux [22], and for reader's convenience, we replicate it here and only make a few minor changes for our problem.

Theorem 5.3. Under the assumptions of Theorem 5.1, the family of processes $\left\{U_{\sigma}(t, \tau)\right\}$, $\sigma \in \Sigma$ corresponding to (5.1) has a bounded (in X) uniformly (w.r.t. $\sigma \in \Sigma$ ) absorbing set $B_{0}$, i.e., there exists a positive constant $\rho$, which depends on $\left\|g_{0}\right\|_{L^{\infty}\left(\mathbb{R} ; L^{2}(\Omega)\right)}$ and the coefficients in (1.6)-(1.8), such that for any bounded subset $B \subset X$ and any $\tau \in \mathbb{R}$, there is a $T=T(B)$ such that for any $t-\tau \geqslant T, \sigma \in \Sigma$ and $\left(u^{0 \tau}, u^{1 \tau}\right) \in B$,

$$
\left\|U_{\sigma}(t, \tau)\left(u^{0 \tau}, u^{1 \tau}\right)\right\|_{X} \leqslant \rho .
$$

Proof. Since $\left\{U_{\sigma}(t, \tau)\right\}, \sigma \in \Sigma$ satisfies the translation identity, we only need to prove Theorem 5.3 for the cases $\tau \equiv 0$. Moreover, from the definition of $\Sigma$ we know that for all $\sigma \in \Sigma$

$$
\|\sigma\|_{L^{\infty}\left(\mathbb{R} ; L^{2}(\Omega)\right)} \leqslant\left\|g_{0}\right\|_{L^{\infty}\left(\mathbb{R} ; L^{2}(\Omega)\right)}
$$

Hence, without loss of generality, in the remainder of proof, we will not point out the difference of symbols and will denote different $\sigma$ by $g$.

For any $\varepsilon \geqslant 0$, we set

$$
E_{\varepsilon}(t)=\frac{1}{2}\|u(t)\|^{2}+\frac{1}{2}\left|u_{t}(t)\right|^{2}+\int_{\Omega} F(u(x)) d x+\varepsilon\left\langle u_{t}(t), u(t)\right\rangle .
$$

Then we have $E_{\varepsilon}(t) \rightarrow E_{0}(t)$ as $\varepsilon \rightarrow 0$. Moreover there exist $C_{0}, C_{1} \geqslant 0$ such that

$$
\frac{C_{0}}{2}\left(\|u(t)\|^{2}+\left|u_{t}(t)\right|^{2}\right)-C_{1} \leqslant E_{0}(t) .
$$

By differentiating (5.4) with time $t$, we obtain that

$$
\begin{aligned}
\frac{d}{d t}\left(E_{\varepsilon}(t)\right) & =\left\langle u_{t t}-\triangle u, u_{t}\right\rangle+\left\langle f(u), u_{t}(t)\right\rangle+\varepsilon\left|u_{t}(t)\right|^{2}+\varepsilon\left\langle u_{t t}(t), u_{t}(t)\right\rangle \\
& =\left\langle g, u_{t}\right\rangle-\left\langle h\left(u_{t}\right), u_{t}\right\rangle+\varepsilon\left|u_{t}(t)\right|^{2}-\varepsilon\|u\|^{2}-\varepsilon\langle f(u), u\rangle+\varepsilon\langle g, u\rangle-\varepsilon\left\langle h\left(u_{t}\right), u\right\rangle .
\end{aligned}
$$


It is obviously that (1.5) implies that

$$
\left\langle h\left(u_{t}\right), u_{t}\right\rangle \geqslant \alpha\left|u_{t}\right|^{2}-C_{2}|\Omega|,
$$

and from (1.7) and (1.8) we know that there are $\lambda_{1}>\delta>0$ and $C>0$ such that

$$
\langle f(u), u\rangle \geqslant \delta \int_{\Omega} F(u) d x-C .
$$

Hence we get the following inequality

$$
\begin{aligned}
E_{\varepsilon}^{\prime}(t) \leqslant & \left(2 \varepsilon-\frac{\alpha}{2}\right)\left|u_{t}(t)\right|^{2}-\frac{\varepsilon}{2}\|u(t)\|^{2}-\varepsilon \delta \int_{\Omega} F(u) d x \\
& -\frac{1}{2}\left\langle h\left(u_{t}\right), u_{t}\right\rangle+\varepsilon\|u(t)\|\left\|h\left(u_{t}(t)\right)\right\|_{H^{-1}}+C,
\end{aligned}
$$

where $C$ depends on $\|g\|_{L^{\infty}\left(\mathbb{R}, L^{2}(\Omega)\right)}$.

On the other hand, from (1.4)-(1.6) we have (e.g., see the Lemma in [22]) that there is a constant $K$ such that

$$
\|h(v)\|_{H^{-1}} \leqslant K(1+\langle h(v), v\rangle) \quad \text { for all } v \in H_{0}^{1}(\Omega) .
$$

Denote

$$
w(t) \triangleq 1+\left\langle h\left(u_{t}(t)\right), u_{t}(t)\right\rangle(>0) .
$$

Then, by taking $\varepsilon$ small enough, we obtain that, for all $t \geqslant 0$,

$$
\begin{aligned}
E_{\varepsilon}^{\prime}(t) & \leqslant-\gamma \varepsilon E_{\varepsilon}(t)+(K \varepsilon\|u(t)\|-1 / 2) w(t)+C \\
& \leqslant-\gamma \varepsilon E_{\varepsilon}(t)+\left(N \varepsilon \sqrt{E_{\varepsilon}(t)}-1 / 2\right) w(t)+C
\end{aligned}
$$

where $N, C>0$ depending only on $f, g, h$ and $\Omega$ (not on the initial data) and $\gamma>0$.

Now choose $\varepsilon>0$ so small that

$$
E_{\varepsilon}(0)<\left(\frac{1}{2 N \varepsilon}\right)^{2}-\frac{C}{\gamma \varepsilon}
$$

Then,

$$
E_{\varepsilon}(t)<\left(\frac{1}{2 N \varepsilon}\right)^{2}, \quad \forall t \geqslant 0
$$

If (5.7) is not true, let $t_{0}=\inf \left\{t \geqslant 0, E_{\varepsilon}(t) \geqslant\left(\frac{1}{2 N \varepsilon}\right)^{2}\right\}$, then $E_{\varepsilon}\left(t_{0}\right)=\left(\frac{1}{2 N \varepsilon}\right)^{2}$ and for all $t \in\left[0, t_{0}\right]$, we have

$$
E_{\varepsilon}(t) \leqslant\left(\frac{1}{2 N \varepsilon}\right)^{2}
$$

Therefore, from (5.6) and (5.8) we can obtain that

$$
E_{\varepsilon}\left(t_{0}\right) \leqslant e^{-\gamma \varepsilon t_{0}} E_{\varepsilon}(0)+\frac{C}{\gamma \varepsilon}<\left(\frac{1}{2 N \varepsilon}\right)^{2} .
$$


This is a contradiction and means that (5.7) is indeed satisfies.

Combining (5.6) and (5.7), by use of the uniform Gronwall lemma, we obtain that

$$
E_{\varepsilon}(t) \leqslant e^{-\gamma \varepsilon t} E_{\varepsilon}(0)+\frac{C}{\gamma \varepsilon}
$$

Finally, we notice that for every bounded set $B \subset X$, assume the bounds of $B$ (in $X$ ) is $E(>0)$, then by taking $1 / \varepsilon=4 N \sqrt{E}$ we can obtain $\frac{1}{(2 N \varepsilon)^{2}}-\frac{C}{\gamma \varepsilon} \geqslant E$ for any $E$ large enough. It follows that there exist $M>0$ (independent of the initial data) and $T=T(B)$ such that

$$
E_{0}(t) \leqslant M\left(1+\sqrt{E_{0}(0)}\right) \text { for all } t \geqslant T \text { and }\left(u^{0 \tau}, u^{1 \tau}\right) \in B .
$$

Without loss of generality, assume $M>1$, then from (5.9) we have that for any bounded set $B \subset X$, there is a $T=T(B)$ such that

$$
E_{0}(t) \leqslant 4 M^{2}+1 \quad \text { for all } t \geqslant T \text { and }\left(u^{0 \tau}, u^{1 \tau}\right) \in B
$$

Combining with (5.5) we know that Theorem [5.3] is true.

\subsection{Uniform (w.r.t. $\sigma \in \Sigma$ ) asymptotic compactness}

The main result in this subsection is summarized in the following theorem.

Theorem 5.4. Let $\Omega$ be a bounded domain in $\mathbb{R}^{3}$ with smooth boundary, and $h$ and $f$ satisfy (1.4)-(1.8). If $g_{0} \in L^{\infty}\left(\mathbb{R} ; L^{2}(\Omega)\right) \cap W_{b}^{1, r}\left(\mathbb{R} ; L^{r}(\Omega)\right)$ and $\Sigma$ is defined by (15.3), then the family of processes $\left\{U_{\sigma}(t, \tau)\right\}, \sigma \in \Sigma$ corresponding to (5.1) or (1.1), is uniformly (w.r.t. $\sigma \in \Sigma$ ) asymptotically compact in $H_{0}^{1}(\Omega) \times L^{2}(\Omega)$.

Hereafter, we always assume that the hypotheses of Theorem 5.1 hold and denote by $B_{0}$ the bounded uniformly absorbing set obtained in Theorem 5.3

\subsubsection{Preliminaries}

Note that condition (1.6) implies that

$$
|h(s)|^{\frac{1}{p}} \leqslant C(1+|s|),
$$

therefore, we have

$$
|h(s)|^{\frac{p+1}{p}}=|h(s)|^{\frac{1}{p}} \cdot|h(s)| \leqslant C(1+|s|)|h(s)| \leqslant C|h(s)|+C h(s) \cdot s .
$$

Combining Young inequality and (1.4) we obtain that

$$
|h(s)|^{\frac{p+1}{p}} \leqslant C(1+h(s) \cdot s) \quad \text { for all } s \in \mathbb{R},
$$


where the constant $C$ is independent of $s$. And from (1.4) and (1.5), we have also that

$$
\frac{C 1}{2} s^{2} \leqslant h(s) s+C \quad \text { for all } s \in \mathbb{R} .
$$

Moreover, we recall the following result.

Lemma 5.5. [19, 24] Let $h$ satisfy (1.4) and (1.5). Then for any $\delta>0$, there exists a constant $C_{\delta}$, depending on $\delta$, such that

$$
|u-v|^{2} \leqslant \delta+C_{\delta}(h(u)-h(v))(u-v) \quad \text { for any } u, v \in \mathbb{R} .
$$

Proposition 5.6. Let $s_{i} \in \mathbb{R}(i=1,2, \cdots)$ and $g \in L^{\infty}\left(\mathbb{R} ; L^{2}(\Omega)\right)$. Then there exists $M>0$ such that

$$
\left\|g\left(x, s_{i}+t\right)\right\|_{L^{2}(\Omega)} \leqslant M \quad \text { for all } t \in \mathbb{R} \backslash \Lambda \text { and all } i=1,2, \cdots,
$$

where $\Lambda \subset \mathbb{R}$ with mes $(\Lambda)=0$ in $\mathbb{R}$.

Proof. Since $g \in L^{\infty}\left(\mathbb{R} ; L^{2}(\Omega)\right)$, we know that there is an $M>0$ such that for each $s_{i}$,

$$
\left\|g\left(x, t+s_{i}\right)\right\|_{L^{2}(\Omega)} \leqslant M \quad \text { for all } t \in \mathbb{R} \backslash \Lambda_{i},
$$

where $\operatorname{mes}\left(\Lambda_{i}\right)=0$ in $\mathbb{R}$.

Then Proposition 5.6 follows immediately by taking $\Lambda=\bigcup_{i=1}^{\infty} \Lambda_{i}$.

Applying Proposition 7.1 of Robinson 34] and Proposition 5.6 above we can deduce the following results immediately:

Proposition 5.7. Let $g \in L^{\infty}\left(\mathbb{R} ; L^{2}(\Omega)\right) \cap W_{b}^{1, r}\left(\mathbb{R} ; L^{r}(\Omega)\right)\left(r>\frac{6}{5}\right)$. Then there is an $M>0$ such that

$$
\sup _{t \in \mathbb{R}}\|g(x, t+s)\|_{L^{2}(\Omega)} \leqslant M \quad \text { for all } s \in \mathbb{R} .
$$

Proposition 5.8. Let $s_{i} \in \mathbb{R}(i=1,2, \cdots)$ and $g \in L^{\infty}\left(\mathbb{R} ; L^{2}(\Omega)\right) \cap W_{b}^{1, r}\left(\mathbb{R} ; L^{r}(\Omega)\right)(r>$ $\left.\frac{6}{5}\right)$. Then there exists $M>0$ such that for any $w \in W_{l o c}^{1,2}\left(\mathbb{R} ; L^{2}(\Omega)\right)$ and any $T>0$,

$$
\begin{aligned}
& \mid \int_{0}^{T} \int_{s}^{T} \int_{\Omega}(\left.g\left(x, \tau+s_{i}\right)-g\left(x, \tau+s_{j}\right)\right) w_{t}(\tau) d x d \tau d s \mid \\
& \leqslant 2 T M\|w(T)\|_{L^{2}(\Omega)}+2 M T^{\frac{1}{2}}\left(\int_{0}^{T} \int_{\Omega}|w(s)|^{2} d x d s\right)^{\frac{1}{2}} \\
& \quad+T \int_{0}^{T} \int_{\Omega}\left|\left(g_{t}\left(x, s+s_{i}\right)-g_{t}\left(x, s+s_{j}\right)\right) w(s)\right| d x d s
\end{aligned}
$$

for all $i, j=1,2, \cdots$. 
Proof. Since

$$
\begin{aligned}
(g(x, t & \left.\left.+s_{i}\right)-g\left(x, t+s_{j}\right)\right) w_{t}(t) \\
\quad & =\frac{d}{d t}\left(\left(g\left(x, t+s_{i}\right)-g\left(x, t+s_{j}\right)\right) w(t)\right)-\left(g_{t}\left(x, t+s_{i}\right)-g_{t}\left(x, t+s_{j}\right)\right) w(t),
\end{aligned}
$$

we have

$$
\begin{aligned}
\left|\int_{0}^{T} \int_{s}^{T} \int_{\Omega}\left(g\left(x, \tau+s_{i}\right)-g\left(x, \tau+s_{j}\right)\right) w_{t}(\tau) d x d \tau d s\right| \\
\leqslant \int_{0}^{T} \int_{\Omega}\left|\left(g\left(x, T+s_{i}\right)-g\left(x, T+s_{j}\right)\right) w(T)\right| d x d s \\
\quad+\int_{0}^{T} \int_{\Omega}\left|\left(g\left(x, s+s_{i}\right)-g\left(x, s+s_{j}\right)\right) w(s)\right| d x d s \\
\quad+\int_{0}^{T} \int_{s}^{T} \int_{\Omega}\left|\left(g_{t}\left(x, \tau+s_{i}\right)-g_{t}\left(x, \tau+s_{j}\right)\right) w(\tau)\right| d x d \tau d s
\end{aligned}
$$

Then by Proposition 5.8 we obtain that for any $T>0$,

$$
\begin{aligned}
\left|\int_{0}^{T} \int_{s}^{T} \int_{\Omega}\left(g\left(x, \tau+s_{i}\right)-g\left(x, \tau+s_{j}\right)\right) w_{t}(\tau) d x d \tau d s\right| \\
\leqslant 2 T M\|w(T)\|_{L^{2}(\Omega)}+2 M \int_{0}^{T}\|w(s)\|_{L^{2}(\Omega)} d s \\
\quad+T \int_{0}^{T} \int_{\Omega}\left|\left(g_{t}\left(x, s+s_{i}\right)-g_{t}\left(x, s+s_{j}\right)\right) w(s)\right| d x d s \\
\leqslant 2 T M\|w(T)\|_{L^{2}(\Omega)}+2 M T^{\frac{1}{2}}\left(\int_{0}^{T} \int_{\Omega}|w(s)|^{2} d x d s\right)^{\frac{1}{2}} \\
\quad+T \int_{0}^{T} \int_{\Omega}\left|\left(g_{t}\left(x, s+s_{i}\right)-g_{t}\left(x, s+s_{j}\right)\right) w(s)\right| d x d s
\end{aligned}
$$

Proposition 5.9. Let $s_{i} \in \mathbb{R}(i=1,2, \cdots), g \in L^{\infty}\left(\mathbb{R} ; L^{2}(\Omega)\right) \cap W_{b}^{1, r}\left(\mathbb{R} ; L^{r}(\Omega)\right)(r>$ $\left.\frac{6}{5}\right),\left\{u_{n}(t) \mid t \geqslant 0, n=1,2, \cdots\right\}$ is bounded in $H_{0}^{1}(\Omega)$, and for any $T_{1}>0,\left\{u_{n_{t}}(t) \mid n=\right.$ $1,2, \cdots\}$ is bounded in $L^{\infty}\left(0, T_{1} ; L^{2}(\Omega)\right)$. Then for any $T>0$, there exist subsequences $\left\{u_{n_{k}}\right\}_{k=1}^{\infty}$ of $\left\{u_{n}\right\}_{n=1}^{\infty}$ and $\left\{s_{n_{k}}\right\}_{k=1}^{\infty}$ of $\left\{s_{n}\right\}_{n=1}^{\infty}$ such that

$$
\lim _{k \rightarrow \infty} \lim _{l \rightarrow \infty} \int_{0}^{T} \int_{s}^{T} \int_{\Omega}\left(g\left(x, \tau+s_{n_{k}}\right)-g\left(x, \tau+s_{n_{l}}\right)\right)\left(u_{n_{k}}-u_{n_{l}}\right)_{t}(\tau) d x d \tau d s=0 .
$$

Proof. Since $\left\{u_{n}(t) \mid t \geqslant 0, n=1,2, \cdots\right\}$ is bounded in $H_{0}^{1}(\Omega)$ and for any $T_{1}>0$, $\left\{u_{n_{t}}(t) \mid n=1,2, \cdots\right\}$ is bounded in $L^{\infty}\left(0, T_{1} ; L^{2}(\Omega)\right)$, then for any $T>0$, without loss of generality (at most by passing subsequence), we assume

$$
u_{n}(T) \rightarrow u_{0} \quad \text { in } \quad L^{2}(\Omega)
$$


and

$$
u_{n} \rightarrow v \quad \text { in } \quad L^{k}\left(0, T ; L^{k}(\Omega)\right)\left(\text { this require } r>\frac{6}{5}\right)
$$

where $k<6$.

Then by use of Hölder inequality and Proposition [5.8, we obtain that

$$
\begin{aligned}
& \lim _{n \rightarrow \infty} \lim _{m \rightarrow \infty} \int_{0}^{T} \int_{s}^{T} \int_{\Omega}\left(g\left(x, \tau+s_{n}\right)-g\left(x, \tau+s_{m}\right)\right)\left(u_{n}-u_{m}\right)_{t}(\tau) d x d \tau d s \\
& \leqslant \lim _{n \rightarrow \infty} \lim _{m \rightarrow \infty} 2 M T\left(\int_{\Omega}\left|u_{n}(T)-u_{m}(T)\right|^{2} d x\right)^{\frac{1}{2}} \\
& \quad+\lim _{n \rightarrow \infty} \lim _{m \rightarrow \infty} 2 M T^{\frac{1}{2}}\left(\int_{0}^{T} \int_{\Omega}\left|u_{n}(s)-u_{m}(s)\right|^{2} d x d s\right)^{\frac{1}{2}} \\
& \quad+\lim _{n \rightarrow \infty} \lim _{m \rightarrow \infty} \int_{0}^{T} \int_{\Omega}\left|\left(g_{t}\left(x, s+s_{n}\right)-g_{t}\left(x, s+s_{m}\right)\right)\left(u_{n}(s)-u_{m}(s)\right)\right| d x d s \\
& =\lim _{n \rightarrow \infty} \lim _{m \rightarrow \infty} T \int_{0}^{T} \int_{\Omega}\left|\left(g_{t}\left(x, s+s_{n}\right)-g_{t}\left(x, s+s_{m}\right)\right)\left(u_{n}(s)-u_{m}(s)\right)\right| d x d s \\
& \leqslant \lim _{n \rightarrow \infty} \lim _{m \rightarrow \infty} T\left(\int_{0}^{T} \int_{\Omega}\left|g_{t}\left(x, s+s_{n}\right)-g_{t}\left(x, s+s_{m}\right)\right|^{r}\right)^{\frac{1}{r}}\left(\int_{0}^{T} \int_{\Omega}\left|u_{n}(s)-u_{m}(s)\right|^{k}\right)^{\frac{1}{k}} \\
& =0 .
\end{aligned}
$$

\subsubsection{A Priori estimates}

The main purpose of this part is to establish (5.19)-(5.21), which will be used to obtain the asymptotic compactness.

For any $\left(u_{0}^{i}, v_{0}^{i}\right) \in B_{0}$, let $\left(u_{i}(t), u_{i_{t}}(t)\right)$ be the corresponding solution to $\sigma_{i}$ with respect to initial data $\left(u_{0}^{i}, v_{0}^{i}\right), i=1,2$, that is, $\left(u_{i}(t), u_{i_{t}}(t)\right)$ is the solution of the following equation

$$
\left\{\begin{array}{l}
u_{t t}+h\left(u_{t}\right)-\Delta u+f(u(t))=\sigma_{i}(x, t) \\
\left.u\right|_{\partial \Omega}=0 \\
\left(u(0), u_{t}(0)\right)=\left(u_{0}^{i}, v_{0}^{i}\right) .
\end{array}\right.
$$

For convenience, we denote

$$
g_{i}(t)=\sigma_{i}(x, t), h_{i}(t)=h\left(u_{i_{t}}(t)\right) \quad t \geqslant 0, \quad i=1,2
$$

and

$$
w(t)=u_{1}(t)-u_{2}(t)
$$


Then $w(t)$ satisfies

$$
\left\{\begin{array}{l}
w_{t t}+h_{1}(t)-h_{2}(t)-\Delta w+f\left(u_{1}(t)\right)-f\left(u_{2}(t)\right)=g_{1}(t)-g_{2}(t) \\
\left.w\right|_{\partial \Omega}=0 \\
\left(w(0), w_{t}(0)\right)=\left(u_{0}^{1}, v_{0}^{1}\right)-\left(u_{0}^{2}, v_{0}^{2}\right)
\end{array}\right.
$$

Set

$$
E_{w}(t)=\frac{1}{2} \int_{\Omega}|w(t)|^{2}+\frac{1}{2} \int_{\Omega}|\nabla w(t)|^{2} .
$$

Step 1. Multiplying (5.13) by $w_{t}(t)$, and integrating over $[s, T] \times \Omega$, we obtain

$$
\begin{aligned}
E_{w}(T) & +\int_{s}^{T} \int_{\Omega}\left(h_{1}(\tau)-h_{2}(\tau)\right) w_{t}(\tau) d x d \tau+\int_{s}^{T} \int_{\Omega}\left(f\left(u_{1}(\tau)\right)-f\left(u_{2}(\tau)\right)\right) w_{t}(\tau) d x d \tau \\
& =\int_{s}^{T} \int_{\Omega}\left(g_{1}(\tau)-g_{2}(\tau)\right) w_{t}(\tau) d x d \tau+E_{w}(s)
\end{aligned}
$$

where $0 \leqslant s \leqslant T$. Then

$$
\begin{aligned}
\int_{s}^{T} \int_{\Omega}\left(h_{1}(\tau)-h_{2}(\tau)\right) w_{t}(\tau) d x d \tau \leqslant & E_{w}(s)+\int_{s}^{T} \int_{\Omega}\left(g_{1}(\tau)-g_{2}(\tau)\right) w_{t}(\tau) d x d \tau \\
& -\int_{s}^{T} \int_{\Omega}\left(f\left(u_{1}(\tau)\right)-f\left(u_{2}(\tau)\right)\right) w_{t}(\tau) d x d \tau
\end{aligned}
$$

Combining with Lemma [5.5, we get that, for any $\delta>0$,

$$
\begin{aligned}
\int_{s}^{T} \int_{\Omega}\left|w_{t}(\tau)\right|^{2} d x d \tau \leqslant & |T-s| \delta \cdot \operatorname{mes}(\Omega)+C_{\delta} E_{w}(s)+C_{\delta} \int_{s}^{T} \int_{\Omega}\left(g_{1}-g_{2}\right) w_{t} d x d \tau \\
& -C_{\delta} \int_{s}^{T} \int_{\Omega}\left(f\left(u_{1}(\tau)\right)-f\left(u_{2}(\tau)\right)\right) w_{t} x d \tau
\end{aligned}
$$

Step 2. Multiplying (5.13) by $w(t)$ and integrating over $[0, T] \times \Omega$, we get that

$$
\begin{aligned}
& \int_{0}^{T} \int_{\Omega}|\nabla w(s)|^{2} d x d s+\int_{\Omega} w_{t}(T) \cdot w(T) d x \\
& =\int_{0}^{T} \int_{\Omega}\left|w_{t}(s)\right|^{2} d x d s-\int_{0}^{T} \int_{\Omega}\left(h_{1}-h_{2}\right) w d x d s+\int_{\Omega} w_{t}(0) \cdot w(0) d x \\
& \quad-\int_{0}^{T} \int_{\Omega}\left(f\left(u_{1}(s)\right)-f\left(u_{2}(s)\right)\right) w d x d s+\int_{0}^{T} \int_{\Omega}\left(g_{1}-g_{2}\right) w d x d s .
\end{aligned}
$$

Therefore, from (5.15) and (5.16), we have

$$
\begin{aligned}
2 \int_{0}^{T} E_{w}(s) d s \leqslant & 2 \delta \operatorname{Tmes}(\Omega)+2 C_{\delta} E_{w}(0)+2 C_{\delta} \int_{0}^{T} \int_{\Omega}\left(g_{1}-g_{2}\right) w_{t} d x d s \\
& -2 C_{\delta} \int_{0}^{T} \int_{\Omega}\left(f\left(u_{1}(s)\right)-f\left(u_{2}(s)\right)\right) w_{t} d x d s-\int_{\Omega} w_{t}(T) w(T)+\int_{\Omega} w_{t}(0) w(0) \\
& -\int_{0}^{T} \int_{\Omega}\left(h_{1}-h_{2}\right) w-\int_{0}^{T} \int_{\Omega}\left(f\left(u_{1}(s)\right)-f\left(u_{2}(s)\right)\right) w+\int_{0}^{T} \int_{\Omega}\left(g_{1}-g_{2}\right) w
\end{aligned}
$$


Integrating (5.14) over $[0, T]$ with respect to $s$, we have that

$$
\begin{aligned}
& T E_{w}(T)+\int_{0}^{T} \int_{s}^{T} \int_{\Omega}\left(h_{1}(\tau)-h_{2}(\tau)\right) w_{t}(\tau) d x d \tau d s \\
& =-\int_{0}^{T} \int_{s}^{T} \int_{\Omega}\left(f\left(u_{1}(\tau)\right)-f\left(u_{2}(\tau)\right)\right) w_{t} d x d \tau d s \\
& \quad+\int_{0}^{T} \int_{s}^{T} \int_{\Omega}\left(g_{1}-g_{2}\right) w_{t} d x d \tau d s+\int_{0}^{T} E_{w}(s) d s \\
& \leqslant-\int_{0}^{T} \int_{s}^{T} \int_{\Omega}\left(f\left(u_{1}(\tau)\right)-f\left(u_{2}(\tau)\right)\right) w_{t} d x d \tau d s+\int_{0}^{T} \int_{s}^{T} \int_{\Omega}\left(g_{1}-g_{2}\right) w_{t} d x d \tau d s \\
& \quad+\delta T m e s(\Omega)+C_{\delta} E_{w}(0)+C_{\delta} \int_{0}^{T} \int_{s}^{T} \int_{\Omega}\left(g_{1}-g_{2}\right) w_{t} d x d s \\
& -C_{\delta} \int_{0}^{T} \int_{\Omega}\left(f\left(u_{1}(s)\right)-f\left(u_{2}(s)\right)\right) w_{t} d x d s-\frac{1}{2} \int_{\Omega} w_{t}(T) w(T)+\frac{1}{2} \int_{\Omega} w_{t}(0) w(0) \\
& \quad-\frac{1}{2} \int_{0}^{T} \int_{\Omega}\left(h_{1}-h_{2}\right) w-\frac{1}{2} \int_{0}^{T} \int_{\Omega}\left(f\left(u_{1}(s)\right)-f\left(u_{2}(s)\right)\right) w+\frac{1}{2} \int_{0}^{T} \int_{\Omega}\left(g_{1}-g_{2}\right) w .
\end{aligned}
$$

Step 3. We need to deal with $\int_{0}^{T} \int_{\Omega}\left(h_{1}-h_{2}\right) w$. Multiplying (5.12) by $u_{i_{t}}(t)$ we obtain

$$
\frac{1}{2} \frac{d}{d t} \int_{\Omega}\left(\left|u_{i_{t}}\right|^{2}+\left|\nabla u_{i}\right|^{2}\right)+\int_{\Omega} h\left(u_{i_{t}}\right) u_{i_{t}}+\int_{\Omega} f\left(u_{i}\right) u_{i_{t}}=\int_{\Omega} g_{i} u_{i_{t}},
$$

which, combining with the existence of bounded uniformly absorbing set, implies that

$$
\int_{0}^{T} \int_{\Omega} h\left(u_{i_{t}}\right) u_{i_{t}} \leqslant M_{T}
$$

where the constant $M_{T}$ depends on $T$ (which is different from the autonomous cases) and the bounds of $B_{0}$. Then, noticing (5.10), we obtain that

$$
\int_{0}^{T} \int_{\Omega}\left|h\left(u_{i_{t}}\right)\right|^{\frac{p+1}{p}} d x d s \leqslant M_{T}
$$

Therefore, using Hölder inequality, from (5.17) we have

$$
\begin{aligned}
\left|\int_{0}^{T} \int_{\Omega} h_{i} w\right| & \leqslant\left(\int_{0}^{T} \int_{\Omega}\left|h\left(u_{i_{t}}\right)\right|^{\frac{p+1}{p}}\right)^{\frac{p}{p+1}}\left(\int_{0}^{T} \int_{\Omega}|w|^{p+1}\right)^{\frac{1}{p+1}} \\
& \leqslant M_{T}^{\frac{p}{p+1}}\left(\int_{0}^{T} \int_{\Omega}|w|^{p+1}\right)^{\frac{1}{p+1}}
\end{aligned}
$$

which implies that

$$
\left|\int_{0}^{T} \int_{\Omega}\left(h_{1}-h_{2}\right) w\right| \leqslant 2 M_{T}^{\frac{p}{p+1}}\left(\int_{0}^{T} \int_{\Omega}|w|^{p+1}\right)^{\frac{1}{p+1}} .
$$

Remark 5.10. To some extent, (5.18) requires that the growth order of $h$ is strictly less than 5 . 
Set

$$
\begin{aligned}
& \phi_{\delta, T}\left(\left(u_{0}^{1}, v_{0}^{1}\right),\left(u_{0}^{2}, v_{0}^{2}\right) ; \sigma_{1}, \sigma_{2}\right) \\
& =-\int_{0}^{T} \int_{s}^{T} \int_{\Omega}\left(f\left(u_{1}(\tau)\right)-f\left(u_{2}(\tau)\right)\right) w_{t} d x d \tau d s \\
& \quad+\left(1+C_{\delta}\right) \int_{0}^{T} \int_{s}^{T} \int_{\Omega}\left(g_{1}-g_{2}\right) w_{t} d x d \tau d s \\
& \quad-C_{\delta} \int_{0}^{T} \int_{\Omega}\left(f\left(u_{1}(s)\right)-f\left(u_{2}(s)\right)\right) w_{t} d x d s-\frac{1}{2} \int_{0}^{T} \int_{\Omega}\left(h_{1}-h_{2}\right) w d x d s \\
& \quad-\frac{1}{2} \int_{0}^{T} \int_{\Omega}\left(f\left(u_{1}(s)\right)-f\left(u_{2}(s)\right)\right) w d x d s+\frac{1}{2} \int_{0}^{T} \int_{\Omega}\left(g_{1}-g_{2}\right) w d x d s, \\
& C_{M}=\delta T m e s(\Omega)+C_{\delta} E_{w}(0)-\frac{1}{2} \int_{\Omega} w_{t}(T) w(T) d+\frac{1}{2} \int_{\Omega} w_{t}(0) w(0) d x .
\end{aligned}
$$

Then we have

$$
E_{w} \leqslant \frac{C_{M}}{T}+\frac{1}{T} \phi_{\delta, T}\left(\left(u_{0}^{1}, v_{0}^{1}\right),\left(u_{0}^{2}, v_{0}^{2}\right) ; \sigma_{1}, \sigma_{2}\right)
$$

\subsubsection{Uniform asymptotic compactness}

\section{Proof of Theorem 5.4;}

Since the family of processes $\left\{U_{\sigma}(t, \tau)\right\}, \sigma \in \Sigma$ has a bounded uniformly absorbing set, for any fixed $\varepsilon>0$, we can choose first $\delta \leqslant \frac{\varepsilon}{2 m e s(\Omega)}$, and then let $T$ so large that

$$
\frac{C_{M}}{T} \leqslant \varepsilon
$$

Hence, thanks to Theorem 4.2, we only need to verify that $\phi_{\delta, T}(\cdot, \cdot ; \cdot, \cdot) \in \mathfrak{C}\left(B_{0}, \Sigma\right)$ for each fixed $T$.

At first, we can observe from the proof procedure of Theorem [5.3 that for any fixed $T$, we have

$$
\bigcup_{\sigma \in \Sigma} \bigcup_{t \in[0, T]} U_{\sigma}(t, 0) B_{0} \text { is bounded in } E_{0}
$$

and the bound depends on $T$.

At the same time, since $m e s_{\mathbb{R}}(\Lambda)=0$, without loss of generality, we assume $T \notin \Lambda$ (or else, taking $T_{1} \notin \Lambda$ satisfies $T_{1}>T$ and replacing $T$ by $T_{1}$ ).

Let $\left(u_{n}, u_{n_{t}}\right)$ be the solutions corresponding to initial data $\left(u_{0}^{n}, v_{0}^{n}\right) \in B_{0}$ with respect to symbol $\sigma_{n} \in \Sigma, n=1,2, \cdots$. Then, from (5.22), without loss of generality (at most by passing subsequence), we assume that

$$
u_{n} \rightarrow u \quad \star-\text { weakly in } L^{\infty}\left(0, T ; L^{6}(\Omega)\right),
$$




$$
\begin{gathered}
u_{n} \rightarrow u \quad \text { in } L^{p+1}\left(0, T ; L^{p+1}(\Omega)\right), \\
u_{n_{t}} \rightarrow u_{t} \quad \star \text {-weakly in } L^{\infty}\left(0, T ; L^{2}(\Omega)\right), \\
u_{n} \rightarrow u \quad \text { in } L^{2}\left(0, T ; L^{2}(\Omega)\right)
\end{gathered}
$$

and

$$
u_{n}(0) \rightarrow u(0) \text { and } u_{n}(T) \rightarrow u(T) \text { in } L^{4}(\Omega),
$$

where we use the compact embeddings $H_{0}^{1} \hookrightarrow L^{4}$ and $H_{0}^{1} \hookrightarrow L^{p+1}$ (since $p<5$ ).

Now, we will deal with each term corresponding to that in (5.19) one by one.

Firstly, from (5.18) we have

$$
\begin{aligned}
\mid \int_{0}^{T} \int_{\Omega}\left(h\left(u_{n_{t}}(s)\right)-\right. & \left.h\left(u_{m_{t}}(s)\right)\right)\left(u_{n}(s)-u_{m}(s)\right) d x d s \mid \\
& \leqslant 2 M_{T}^{\frac{p}{p+1}}\left(\int_{0}^{T} \int_{\Omega}\left|u_{n}(s)-u_{m}(s)\right|^{p+1}\right)^{\frac{1}{p+1}}
\end{aligned}
$$

where $M_{T}$ depends on $T$ and the norm of $B_{0}$ in $H_{0}^{1} \times L^{2}$. Therefore, from (5.24) we can get

$$
\lim _{n \rightarrow \infty} \lim _{m \rightarrow \infty} \int_{0}^{T} \int_{\Omega}\left(h\left(u_{n_{t}}(s)\right)-h\left(u_{m_{t}}(s)\right)\right)\left(u_{n}(s)-u_{m}(s)\right) d x d s=0 .
$$

Secondly, from Proposition [5.7] and (5.27), by the similar method used in the proof of Proposition 5.8, we can obtain that

$$
\lim _{n \rightarrow \infty} \lim _{m \rightarrow \infty} \int_{0}^{T} \int_{\Omega}\left(g_{n}(x, s)-g_{m}(x, s)\right)\left(u_{n_{t}}(s)-u_{m_{t}}(s)\right) d x d s=0,
$$

and from Proposition 5.9 we can get that

$$
\lim _{n \rightarrow \infty} \lim _{m \rightarrow \infty} \int_{0}^{T} \int_{s}^{T} \int_{\Omega}\left(g_{n}(x, \tau)-g_{m}(x, \tau)\right)\left(u_{n_{t}}(\tau)-u_{m_{t}}(\tau)\right) d x d \tau d s=0 .
$$

At the same time, from the growth condition (1.7) and (5.26), we can get easily that

$$
\lim _{n \rightarrow \infty} \lim _{m \rightarrow \infty} \int_{0}^{T} \int_{\Omega}\left(f\left(u_{n}(s)\right)-f\left(u_{m}(s)\right)\right)\left(u_{n}(s)-u_{m}(s)\right) d x d s=0 .
$$


Finally, since

$$
\begin{aligned}
\int_{0}^{T} & \int_{\Omega}\left(u_{n_{t}}(s)-u_{m_{t}}(s)\right)\left(f\left(u_{n}(s)\right)-f\left(u_{m}(s)\right)\right) d x d s \\
= & \int_{0}^{T} \int_{\Omega} u_{n_{t}} f\left(u_{n}(s)\right)+\int_{0}^{T} \int_{\Omega} u_{m_{t}} f\left(u_{m}(s)\right) \\
& \quad-\int_{0}^{T} \int_{\Omega} u_{n_{t}} f\left(u_{m}(s)\right)-\int_{0}^{T} \int_{\Omega} u_{m_{t}} f\left(u_{n}(s)\right) \\
= & \int_{\Omega} F\left(u_{n}(T)\right)-\int_{\Omega} F\left(u_{n}(0)\right)+\int_{\Omega} F\left(u_{m}(T)\right)-\int_{\Omega} F\left(u_{m}(0)\right) \\
& \quad-\int_{0}^{T} \int_{\Omega} u_{n_{t}} f\left(u_{m}(s)\right)-\int_{0}^{T} \int_{\Omega} u_{m_{t}} f\left(u_{n}(s)\right),
\end{aligned}
$$

then, by use of (5.23), (5.25), (5.27) and (1.7), taking first $m \rightarrow \infty$, then $n \rightarrow \infty$, we obtain that

$$
\begin{aligned}
\lim _{n \rightarrow \infty} & \lim _{m \rightarrow \infty} \int_{0}^{T} \int_{\Omega}\left(u_{n_{t}}(s)-u_{m_{t}}(s)\right)\left(f\left(u_{n}(s)\right)-f\left(u_{m}(s)\right)\right) d x d s \\
= & \int_{\Omega} F(u(T))-\int_{\Omega} F(u(0))+\int_{\Omega} F(u(T))-\int_{\Omega} F(u(0)) \\
& \quad-\int_{0}^{T} \int_{\Omega} u_{t} f(u(s))-\int_{0}^{T} \int_{\Omega} u_{t} f(u(s)) \\
= & 0 .
\end{aligned}
$$

Similarly, we have

$$
\begin{gathered}
\int_{s}^{T} \int_{\Omega}\left(u_{n_{t}}(\tau)-u_{m_{t}}(\tau)\right)\left(f\left(u_{n}(\tau)\right)-f\left(u_{m}(\tau)\right)\right) d x d \tau \\
=\int_{\Omega} F\left(u_{n}(T)\right)-\int_{\Omega} F\left(u_{n}(s)\right)+\int_{\Omega} F\left(u_{m}(T)\right)-\int_{\Omega} F\left(u_{m}(s)\right) \\
\quad-\int_{s}^{T} \int_{\Omega} u_{n_{t}} f\left(u_{m}(\tau)\right)-\int_{s}^{T} \int_{\Omega} u_{m_{t}} f\left(u_{n}(\tau)\right) .
\end{gathered}
$$

At the same time, $\left|\int_{s}^{T} \int_{\Omega}\left(u_{n_{t}}(\tau)-u_{m_{t}}(\tau)\right)\left(f\left(u_{n}(\tau)\right)-f\left(u_{m}(\tau)\right)\right) d x d \tau\right|$ is bounded for each fixed $T$, then by Lebesgue dominated convergence theorem we have

$$
\begin{aligned}
& \lim _{n \rightarrow \infty} \lim _{m \rightarrow \infty} \int_{0}^{T} \int_{s}^{T} \int_{\Omega}\left(u_{n_{t}}(\tau)-u_{m_{t}}(\tau)\right)\left(f\left(u_{n}(\tau)\right)-f\left(u_{m}(\tau)\right)\right) d x d \tau d s \\
& =\int_{0}^{T}\left(\lim _{n \rightarrow \infty} \lim _{m \rightarrow \infty} \int_{s}^{T} \int_{\Omega}\left(u_{n_{t}}(\tau)-u_{n_{t}}(\tau)\right)\left(f\left(u_{n}(\tau)\right)-f\left(u_{m}(\tau)\right)\right) d x d \tau\right) d s \\
& =\int_{0}^{T} 0 d s=0 .
\end{aligned}
$$

Hence, combining (5.28)-(15.33), we get that $\phi_{\delta, T}(\cdot, \cdot ; \cdot, \cdot) \in \mathfrak{C}\left(B_{0}, \Sigma\right)$, and then this completes the proof of Theorem [5.4] 
Remark 5.11. If $g_{0} \in L^{\infty}\left(\mathbb{R} ; L^{2}(\Omega)\right) \cap L_{c}^{2}\left(\mathbb{R} ; L^{2}(\Omega)\right)$ (e.g., $g_{0} \in L^{\infty}\left(\mathbb{R} ; L^{2}(\Omega)\right.$ ) and is a time periodic, quasi-periodic or almost periodic function in $L_{\text {loc }}^{2}\left(\mathbb{R} ; L^{2}(\Omega)\right)$ ), then we can obtain (5.28) and (5.29) directly from the definition of $L_{c}^{2}\left(\mathbb{R} ; L^{2}(\Omega)\right)$. That is, we do not need all of the preliminaries from Proposition [5.6 to Proposition [5.9, and Theorem 5.4 on uniform asymptotic compactness still holds.

\subsection{Existence of uniform attractor}

Theorem 5.12. Let $\Omega$ be a bounded domain in $\mathbb{R}^{3}$ with smooth boundary, and $h$ and $f$ satisfy (1.4) -(1.8). If $g_{0} \in L^{\infty}\left(\mathbb{R} ; L^{2}(\Omega)\right) \cap W_{b}^{1, r}\left(\mathbb{R} ; L^{r}(\Omega)\right)$ and $\Sigma$ is defined by (5.3), then the family of processes $\left\{U_{\sigma}(t, \tau)\right\}, \sigma \in \Sigma$ corresponding to (5.1) or (1.1) has a compactly uniform (w.r.t. $\sigma \in \Sigma$ ) attractor $\mathscr{A}_{\Sigma}$.

Proof. From Theorem 5.3 and Theorem 5.4 we know that the conditions of Theorem 3.4 are all satisfied.

Remark 5.13. If $g_{0} \in L^{\infty}\left(\mathbb{R} ; L^{2}(\Omega)\right)$ and $g_{0}$ is a time periodic, quasi-periodic or almost periodic function in $L_{\text {loc }}^{2}\left(\mathbb{R} ; L^{2}(\Omega)\right)$, then the family of processes $\left\{U_{\sigma}(t, \tau)\right\}, \sigma \in \Sigma$ corresponding to (5.1) or (1.1) has a compactly uniform (w.r.t. $\sigma \in \Sigma$ ) attractor $\mathscr{A}_{\Sigma}$.

\subsection{Structure of uniform attractor}

In this subsection, we will consider the structure of a uniform attractor by applying Theorem 3.8 and Theorem 3.10

For this purpose, we need some continuities for the processes, and then we need to assume some additional conditions on the external term $g$, since we need to know whether $\Sigma$ with the $*$-weak topology of $L^{\infty}\left(\mathbb{R} ; L^{2}(\Omega)\right) \cap W_{b}^{1, r}\left(\mathbb{R} ; L^{2}(\Omega)\right)$ is metrizable and when it becomes a compact metric space.

We assume that $g_{0} \in W^{1, \infty}\left(\mathbb{R} ; L^{2}(\Omega)\right)$, and set

$$
\Sigma_{0}^{\prime}=\left\{g_{0}(x, t+h) \mid h \in \mathbb{R}\right\}
$$

and

$$
\Sigma^{\prime} \text { be the } * \text {-weakly closure of } \Sigma_{0}^{\prime} \text { in } W^{1, \infty}\left(\mathbb{R} ; L^{2}(\Omega)\right) .
$$

Then, by the classical results (e.g., see [17]), we see that $\Sigma^{\prime}$ with the $*$-weak topology of $W^{1, \infty}\left(\mathbb{R} ; L^{2}(\Omega)\right)$ forms a sequentially compact and metrizable space. We denote the equivalent metric by $d_{1}(\cdot, \cdot)$. Thus $\left(\Sigma^{\prime}, d_{1}\right)$ is a compact metric space.

In order to prove the norm-to-weak continuity later, we also need the following simple property. 
Proposition 5.14. Let $u_{n} \rightarrow u$ in $L^{2}\left(0, T ; H_{0}^{1}(\Omega)\right)$ and $u_{n_{t}} \rightarrow u_{t}$ in $L^{2}\left(0, T ; L^{2}(\Omega)\right)$. Then $u_{n}(t) \rightarrow u(t)$ in $L^{2}(\Omega)$ for all $t \in[0, T]$.

This can be proved by a simple application of Proposition 7.1 and Theorem 8.1 of Robinson 34, and thus we omit it here. Moreover, we have the following observation.

Proposition 5.15. The translation semigroup $\{T(t)\}_{t \geqslant 0}$ is invariant and continuous in $\Sigma^{\prime}$ with respect to the $*$-weak topology of $W^{1, \infty}\left(\mathbb{R} ; L^{2}(\Omega)\right)$, equivalently, with respect to the metric $d_{1}$.

Lemma 5.16. The family of processes $\left\{U_{\sigma}(t, \tau)\right\}, \sigma \in \Sigma^{\prime}: X \times \Sigma^{\prime} \mapsto X$ is norm-toweak continuous.

Proof. Without loss of generality, we take $\tau=0$.

Multiplying (5.12) by $u_{t}$ and integrating over $[0, t] \times \Omega$, then from Theorem 5.3 we can obtain that

$$
\sup _{t \geqslant 0}\left\|U_{\sigma}(t, 0) y_{0}\right\|_{X} \leqslant c\left(\left\|y_{0}\right\|_{X}\right) \quad \forall \sigma \in \Sigma^{\prime}, y_{0} \in X
$$

where $c(\cdot)$ is a monotone increasing function on $\mathbb{R}^{+}$.

Let $y_{n} \rightarrow y_{0}$ in $X, \sigma_{n} \in \Sigma^{\prime}$ and $\sigma_{n} \rightarrow \sigma$ with respect to metric $d_{1}$. Set $\left(u_{n}(t), u_{n_{t}}(t)\right)=$ $U_{\sigma_{n}}(t, 0) y_{n}$ and $\left(u(t), u_{t}(t)\right)=U_{\sigma}(t, 0) y_{0}$.

Then from (5.36) we know that $\left\{U_{\sigma_{n}}(t, 0) y_{n}\right\}$ is bounded in $L^{\infty}(0, T ; X)$, and due to the growth condition (1.7) we also have that $\left\{f\left(u_{n}(t)\right)\right\}$ is bounded in $L^{\infty}\left(0, T ; L^{2}(\Omega)\right)$. At the same time, since $u_{n}(t)$ is the solution of (5.12) we can deduce that $\left\{u_{n_{t t}}\right\}$ is also bounded in $L^{\infty}\left(0, T ; H^{-1}(\Omega)\right)$. Therefore, there exist subsequence $u_{n_{k}}$ such that

$$
\begin{gathered}
\left(u_{n_{k}}(t), u_{n_{k_{t}}}(t)\right) \rightarrow\left(\tilde{u}(t), \tilde{u}_{t}(t)\right) \quad \text { weakly in } L^{2}(0, T ; X), \\
f\left(u_{n_{k}}\right) \rightarrow \chi \quad * \text {-weakly in } L^{\infty}\left(0, T ; L^{2}(\Omega)\right), \\
u_{n_{k_{t t}}} \rightarrow \tilde{u}_{t t}(t) \quad \text { weakly in } L^{2}\left(0, T ; H^{-1}(\Omega)\right) .
\end{gathered}
$$

Similar to that in Lions [27, noticing that $u_{n_{k}}(t)$ is bounded in $H_{0}^{1}(\Omega)$, which implies that $u_{n_{k}}(t)$ has a subsequence (here we also denote it by $u_{n_{k}}(t)$ ) convergent to $\tilde{u}(t)$ almost everywhere on $\Omega$, we can get $\chi=f(\tilde{u}(t))$. Hence, $\tilde{u}(t)$ is a solution corresponding to initial data $y_{0}$ with respect to symbol $\sigma$. Then, by the uniqueness of solution we have that $\tilde{u}=u$. 
From (5.37) and Proposition 5.14 we have that $u_{n_{k}}(t) \rightarrow u(t)$ in $L^{2}(\Omega)$ for all $t \in[0, T]$. On the other hand, from (5.36) we know that $\left\{u_{n_{k}}(t)\right\}$ is bounded in $H_{0}^{1}(\Omega)$, therefore, $u_{n_{k}}(t) \rightarrow u(t)$ in $H_{0}^{1}(\Omega)$ for all $t \in[0, T]$. Hence, for all $t \in[0, T]$, we have $u_{n}(t) \rightarrow u(t)$ in $H_{0}^{1}(\Omega)$.

Similarly, from (5.37), (5.39) and the fact that $\left\{u_{n_{t t}}\right\}$ is bounded in $L^{\infty}\left(0, T ; H^{-1}(\Omega)\right)$, we have that $u_{n_{t}}(t) \rightarrow u_{t}(t)$ in $L^{2}(\Omega)$ for any $t \in[0, T]$.

Applying Theorem 3.10, from Theorem 5.3, Theorem 5.4, Proposition 5.5]and Lemma 5.16. we deduce the following result.

Theorem 5.17. Let $\Omega$ be a bounded domain in $\mathbb{R}^{3}$ with smooth boundary, and $h$ and $f$ satisfy (1.4) -(1.8). If $g_{0} \in W^{1, \infty}\left(\mathbb{R} ; L^{2}(\Omega)\right)$ and $\Sigma^{\prime}$ is defined by (5.35), then the family of processes $\left\{U_{\sigma}(t, \tau)\right\}, \sigma \in \Sigma^{\prime}$ corresponding to (5.1) has a compactly uniform (w.r.t. $\left.\sigma \in \Sigma^{\prime}\right)$ attractor $\mathscr{A}_{\Sigma^{\prime}}$. Moreover,

$$
\mathscr{A}_{\Sigma_{0}^{\prime}}=\mathscr{A}_{\Sigma^{\prime}}=\bigcup_{\sigma \in \Sigma^{\prime}} \mathcal{K}_{\sigma}(0)
$$

\section{References}

[1] J. Arrietta, A.N. Carvalho and J.K. Hale, A damped hyperbolic equations with critical exponents, Comm. Partial Differential Equations, 17(1992) 841-866.

[2] J.M. Ball, Global attractors for damped semilinear wave equations, Discrete Contin. Dyn. Syst., 10(2004) 31-52.

[3] A.V. Babin and M.I. Vishik, Attractors of Evolution Equations, NorthHolland, Amsterdam, 1992.

[4] V. P. Bongolan-Walsh, D. Cheban and J. Duan, Recurrent Motions in the Nonautonomous Navier-Stokes System, Discrete Contin. Dyn. Syst. B, 3(2002) 255-262.

[5] T. Caraballo, P.E. Kloeden and J. Real, Pullback and forward attractors for a damped wave equation with delays, Stochastics and Dynamics, 4(2004) 405-423.

[6] D.N. Cheban and J. Duan, Almost periodic motions and global attractors of the nonautonomous Navier-Stokes e quations, J. Dyn. Differential Equations, 16(2004), 1-34.

[7] V.V. Chepyzhov and A. Miranville, Trajectory and global attractors of dissipative hyperbolic equations with memory, Commun. Pure Appl. Anal., 4(2005) 115-142.

[8] V.V. Chepyzhov and M.I. Vishik, A Hausdorff dimension estimate for kernel sections of non-autonomous evolution equations, Indiana Univ. Math. J., 42(1993) 1057-1076. 
[9] V.V. Chepyzhov and M.I. Vishik, Attractors of nonautonomous dynamical systems and their dimension, J. Math. Pures Appl., 73(1994) 279-333.

[10] V.V. Chepyzhov and M.I. Vishik, Non-autonomous evolutionary equations with translationcompact symbols and their attractors, C. R. Acad. Sci. Paris Sér. I, 321(1995) 153-158.

[11] V.V. Chepyzhov and M.I. Vishik, Attractors for equations of mathematical physics, Amer. Math. Soc. Colloq. Publ., Vol. 49, Amer. Math. Soc., Providence, RI, 2002.

[12] I. Chueshov and I. Lasiecka, Attractors for second-order evolution equations with nonlinear damping, J. Dyn. Differential Equations, 16(2004) 469-512.

[13] I. Chueshov and I. Lasiecka, Long-time behavior of second order evolution equations with nonlinear damping, preprint, 2004.

[14] I. Chueshov and I. Lasiecka, Long time dynamics of semilinear wave equation with nonlinear interior-boundary damping and sources of critical exponents, preprint.

[15] I. Chueshov and I. Lasiecka, Long-time Dynamics of von Karman Semi-flows with Nonlinear boundary/interior Damping, preprint.

[16] L. Chierchia and J. You, KAM tori for 1D wave equations with periodic boundary conditions, Comm. Math. Phys., 211(2000) 497-525.

[17] J. Diestel, Sequences and series in Banach Spaces, Springer-Verlag, GTM 92, 1984.

[18] A. Eden, V. Kalantarov and A. Miranville, Finite-dimensional attractors for a general class of nonautonomous wave equations, Appl. Math. Lett., 13(2000) 17-22.

[19] E. Feireisl, Global attractors for damped wave equations with supercritical exponent, J. Differential Equations, 116(1995) 431-447.

[20] J.M. Ghidagla and A. Marzocchi, Longtime behaviour of strongly damped wave equations, global attractors and their dimension, SIAM J. Math. Anal., 22(1991) 879-895.

[21] J.K. Hale, Asymptotic Behavior of Dissipative Systems, Amer. Math. Soc., Providence, RJ, 1988.

[22] A. Haraux, Recent results on semilinear wave equations with dissipation, Pitman Research Notes in Math., 141(1986) 150-157.

[23] A. Haraux, Systemes dynamiques dissipatifs et applications, Paris, Masson, 1991.

[24] A.Kh. Khanmamedov, Global attractors for von Karman equations with nonlinear interior dissipation, J. Math. Anal. Appl., 318(2006) 92-101.

[25] A.Kh. Khanmamedov, Existence of a global attractor for the plate equation with a critical exponent in an unbounded domain, Applied Mathematics Letters, 18(2005) 827-832. 
[26] O.A. Ladyzhenskaya, Attractors for semigroups and evolution equations, Leizioni Lincei, Cambridge University Press, Cambridge, New York, 1991.

[27] J.L. Lions, Quelques methodes de resolution des problemes aux limites nlineaires, Dunod, Paris, 1969.

[28] G. Lukaszewicz and W. Sadowski, Uniform attractor for 2D magneto-micropolar fluid flow in some unbounded domains, Z. Angew. Math. Phys., 55(2004) 1-11.

[29] S.S. Lu, H.Q. Wu and C.K. Zhong, Attractors for nonautonomous $2 D$ Navier-Stokes equations with normal external forces, Discrete Contin. Dyn. Syst., 13(2005) 701-719.

[30] Q.F. Ma, S.H. Wang and C.K. Zhong, Necessary and sufficient conditions for the existence of global attractors for semigroups and applications, Indiana University Math. J., 51(2002) 1541-1557.

[31] A. Majda, Introduction to PDEs and Waves for the Atmosphere and Oceans, American Math. Soc., Providence, RI, 2003.

[32] I. Moise, R. Rosa and X. Wang, Attractors for noncompact nonautonomous systems via energy equations, Discrete Contin. Dyn. Syst., 10(2004) 473-496.

[33] I. Moise, R. Rosa and X. Wang, Attractors for non-compact semigroup via energy equations, Nonlinearity, 11(1998) 1369-1393.

[34] J.C. Robinson, Infinite-dimensional dynamical systems, An introduction to dissipative parabolic PDEs and the theory of global attractors, Cambridge University Press 2001.

[35] G.R. Sell and Y. You, Dynamics of evolutionary equations, Springer, New York, 2002.

[36] C.Y. Sun, M.H. Yang and C.K. Zhong, Global attractors for the wave equation with nonlinear damping, J. Differential Equations, 227(2006) 427-443.

[37] C.Y. Sun, M.H. Yang and C.K. Zhong, Global attractors for hyperbolic equations with critical exponent in locally uniform spaces, submitted.

[38] R. Temam, Infinite-Dimensional Dynamical Systems in Mechanics and Physics, Springer, New York, 1997.

[39] S.V. Zelik, Asymptotic regularity of solutions of a nonautonomous damped wave equation with a critial growth exponent, Comm. Pure Appl. Anal., 4(2004) 921-934.

[40] C.K. Zhong, M.H. Yang and C.Y. Sun, The existence of global attractors for the norm-toweak continuous semigroup and application to the nonlinear reaction-diffusion equations, J. Differential Equations, 223(2006) 367-399.

[41] S.F. Zhou and L.S. Wang, Kernel sections for damped non-autonomous wave equations with critical exponent, Discrete Contin. Dyn. Syst., 9(2003) 399-412. 